Classification

Physics Abstracts

$42.70-44.40-44.50-86.10 \mathrm{~K}$

\title{
L'imagerie ultrasonore
}

\author{
M. Fink \\ Laboratoire d'Acoustique Moléculaire, Université Louis Pasteur, 67000 Strasbourg, France
}

(Reçu le 17 mai 1983, révisé le 2 juin, accepté le 6 juin 1983)

\begin{abstract}
Résumé. - Parallèlement au développement des nouvelles techniques d'imagerie (scanner à rayons $\mathrm{X}$, résonance magnétique nucléaire, radar à ouverture synthétique...), les applications de l'imagerie ultrasonore connaissent des progrès spectaculaires. Après un rappel des principes de l'imagerie ultrasonore, on propose une revue synthétique de la recherche dans ce domaine. Une comparaison des succès obtenus en diagnostic médical, en contrôle non destructif et en imagerie sous-marine est présentée. On met en évidence l'originalité d'un certain nombre de techniques d'imagerie ultrasonore relativement aux techniques de l'imagerie optique. On décrit en particulier les nouvelles techniques de reconstruction tomographique en mode échographique qui sont appelées à un grand essor. C'est l'utilisation de techniques de traitement du signal sophistiquées qui permet cette nouvelle approche en échographie et qui doit déboucher d'ici peu sur une imagerie « quantitative » des paramètres acoustiques.
\end{abstract}

\begin{abstract}
Simultaneously to the development of new imaging techniques (X-ray scanner, NMR imaging, synthetic aperture radar...) spectacular progress in ultrasound imaging has been made in recent years. Following a summary of imaging ultrasound principles, this article is a review of the main attempts performed in this research area. An evaluation of the various ultrasound techniques is done in the fields of medical diagnosis, non destructive testing and underwater imaging. After a comparison between these various techniques, the emphasis is put on the particularities of ultrasound imaging techniques. The new area of echotomography is described. The use of sophisticated signal processing techniques enables this new approach in echography, which must lead soon to a quantitative " imaging of acoustical parameters.
\end{abstract}

- Depuis une dizaine d'années, les applications de l'imagerie ultrasonore au diagnostic médical ont connu un grand développement. Les procédés de l'holographie acoustique, si prometteurs au départ, ont été complètement abandonnés dans ce domaine, et ce sont des techniques d'échographie qui dominent le marché médical actuel. Parallèlement au développement des "scanners" à rayons $X$, de nouveaux procédés d'imagerie ultrasonore sont étudiés depuis un certain temps (reconstruction tomographique en transmission ou en échographie assistée par ordinateur). Après avoir dans un premier temps cherché à transférer avec peu de succès les techniques du " scanner " dans le domaine ultrasonore, les chercheurs s'orientent maintenant vers des techniques de reconstruction tomographique typiquement ultrasonore (technique du temps de vol et surtout tomographie en mode échographique).

- Parallèlement à ces recherches en imagerie médicale, se sont développés en imagerie sous-marine à faible profondeur $(2 \mathrm{à} 100 \mathrm{~m})$ des procédés variés : focalisation échographique, synthèse de faisceau (beam forming), caméra ultrasonore équipée de lentilles acoustiques, dispositifs nombreux d'holographie acoustique assistés par ordinateurs.

- A l'heure actuelle la diversité des techniques proposées dans le domaine des applications médicales et dans celui des applications sous-marines semble beaucoup plus vaste que celle qui est retenue dans le domaine du contrôle non destructif.

- A première vue, on peut espérer que cette avance technologique puisse être transférée dans le domaine du contrôle non destructif. Il y a cependant des différences fondamentales entre ces types d'applications.

- La possibilité de former de bonnes images de structures biologiques en utilisant les ultrasons comme source d'information est liée à un certain nombre de conditions favorables qui se trouvent réunies dans les tissus biologiques. En effet, la plupart des tissus autres que l'os ont une densité $\rho_{0}$ voisine de celle de l'eau et la vitesse de propagation des ondes ultrasonores dans ces milieux est pratiquement constante $\left(c=1500 \mathrm{~m} \cdot \mathrm{s}^{-1}\right)$. Cette relative uniformité des propriétés acoustiques des tissus à étudier explique la possibilité d'une exploration précise en profondeur. 
Entre deux tissus, la faible différence d'impédance acoustique $\left(Z=\rho_{0} c\right)$ est telle que l'écho renvoyé par l'interface (Fig. 1) est facilement détectable alors que la majeure partie de l'onde est transmise et peut servir à une exploration en aval des organes examinés.

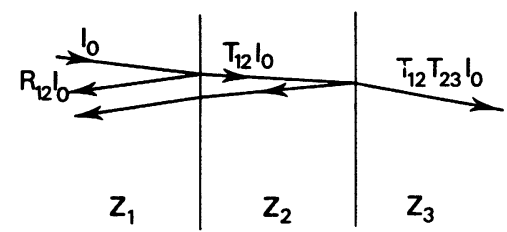

Fig. 1. - Réflexion et transmission d'une onde ultrasonore à l'interface de matériaux d'impédance $Z_{1}, Z_{2}$ et $Z_{3}$.

[Ultrasound reflection and transmission through parallel layers of different impedances.]

En se limitant à des fréquences ultrasonores de quelques $\mathrm{MHz}$, pour lesquelles l'atténuation dans les tissus n'est pas trop importante, il est alors possible d'accéder à une information très riche sur la position des nombreuses interfaces biologiques qui existent sur une profondeur d'exploration qui peut atteindre une vingtaine de centimètres.

La densité d'information obtenue est alors extrêmement importante, et lorsque l'image d'une coupe des organes est présentée sous la forme d'une carte bidimensionnelle qui module, par exemple, l'intensité d'un moniteur de télévision, elle nécessite de la part du cerveau, comme de l'œil du médecin, un traitement de reconnaissance de forme très sophistiquée. Il faut en particulier pouvoir reconnaître parmi les contours et à l'intérieur des organes, certaines anomalies qui sont souvent fortement masquées par la grande quantité d'informations provenant des régions saines.

- Le type de milieu observé en contrôle non destructif est totalement différent. On cherche à repérer au milieu d'un matériau homogène la présence de défauts (fissures, inclusions) généralement très peu nombreux. On veut préciser la position, la taille et l'orientation de ces défauts, afin de pouvoir décider si la pièce examinée risque de se détériorer rapidement. Dans les métaux, comme dans les céramiques, la présence de ces défauts se manifeste par de très fortes ruptures d'impédance acoustique localisées dans des zones relativement petites. Les perturbations du champ ultrasonore traversant ces milieux seront très fortes et en outre contrairement au cas des milieux quasiliquides que sont les tissus biologiques, les modes d'ondes ultrasonores qui peuvent se propager ne seront plus uniquement du type longitudinal, mais aussi des modes de cisaillement et des modes de surface (onde de Rayleigh, de Lamb, etc.). Une telle situation nécessite un examen précis du type d'onde à émettre, afin d'éviter si possible les conversions de modes qui peuvent prendre naissance aux interfaces et qui peuvent dégrader fortement l'efficacité des procédés d'imagerie.
- Une autre différence fondamentale avec l'imagerie médicale vient de ce que la région de l'objet qui nous intéresse est généralement petite (de l'ordre de quelques longueurs d'onde). Les techniques d'imagerie ultrasonore, qui sont limitées en résolution par la longueur d'onde et par la dimension des transducteurs, sont alors un peu rustiques et elles ne permettent pas de préciser correctement la forme et la taille des défauts. On est alors tenté, plutôt que de réaliser une image de l'objet de façon classique (déplacement d'un transducteur focalisé, holographie, lentille, etc.), d'étudier avec soin la répartition de l'énergie diffusée par le défaut en fonction de l'angle et de la fréquence ultrasonore. Cette étude de la " signature » du défaut, défaut qui dans un premier temps a été repéré par un dispositif d'imagerie classique, peut alors permettre en utilisant des algorithmes de reconstruction sophistiqués de remonter à sa forme et à sa taille. Ce domaine d'activité qui est en fait une extension des techniques d'imagerie, au-delà des limites classiques de la résolution, nécessite une parfaite maîtrise des modèles de propagation ultrasonores dans les solides en présence d'inhomogénéités, en tenant compte de tous les types d'ondes qui se propagent dans de tels milieux. C'est une recherche qui a fait des progrès importants ces dernières années.

- On peut rattacher à cette démarche, le domaine de la caractérisation des tissus par ultrasons. On cherche là aussi, par une analyse très précise des échos renvoyés par une tranche de tissu, à compléter par des informations quantitatives, les images ultrasonores fournies aux médecins. Une étude précise du rayonnement ultrasonore diffusé en fonction de l'angle et de la fréquence peut permettre de remonter à la microstructure et à l'architectưre des tissus.

\section{Les principes de l'imagerie ultrasonore.}

Après cette introduction sur les différences existant entre le contrôle non destructif et le diagnostic médical, nous allons présenter la démarche qui, jusqu'à présent, est systématiquement suivie dans pratiquement toutes les expériences d'imagerie. Il faut pour cela éclaircir la façon dont interagit un faisceau d'ultrasons avec un milieu inhomogène. On verra alors que dans les 2 cas du contrôle non destructif et du diagnostic médical, et pour des raisons bien différentes, le problème de la réalisation de l'image acoustique se ramène à celui du développement d'un procédé de reconnaissance de formes où la forme élémentaire à reconnaître est celle d'un front d'onde sphérique. De très nombreux procédés de décodage de ces ondes sphériques ont été développés : lentilles acoustiques, holographie acoustique, focalisation échographique par lignes à retard, focalisation par déphasage, reconstruction tomographique en réflexion, ouverture synthétique à focalisation digitale, etc. Dans toutes ces techniques d'imagerie, on fait l'hypothèse que pendant l'illumination acoustique de l'objet, 
on ne génère qu'un seul type de mode d'onde ultrasonore. C'est naturellement le cas en imagerie médicale, où seules les ondes longitudinales peuvent se propager dans les tissus biologiques. C'est aussi le cas en imagerie sous-marine, avec cependant comme restriction la possibilité d'apparition d'ondes de surface et de cisaillement au niveau des cibles visualisées.

- En contrôle non destructif, par contre, on peut utiliser à l'émission soit des ondes longitudinales, soit des ondes de cisaillement, soit des ondes de surface type Rayleigh, et on fera l'hypothèse qu'aucune conversion de mode n'a lieu au cours de l'interaction de l'onde incidente avec la matière examinée. C'est bien évidemment une hypothèse très restrictive qui est mise en défaut dans le cas de la visualisation de certaines structures échogènes. On ne peut alors, si l'on ne dispose d'aucune connaissance a priori sur la forme du défaut, en faire son image.

- L'hypothèse de la propagation d'un seul type de mode acoustique permet d'adopter comme modèle celui d'un champ de pression acoustique $p(\mathbf{r}, t)$ vérifiant, pour un milieu homogène de célérité $c$ l'équation d'onde

$$
\square p(\mathbf{r}, t)=0 \quad \text { où } \quad \square=\Delta-\frac{1}{c^{2}} \frac{\partial^{2}}{\partial t^{2}} .
$$

La présence d'un certain nombre d'inhomogénéités associées soit aux tissus biologiques, soit aux défauts présents dans les matériaux, va modifier cette équation d'onde en introduisant un ensemble de termes sources qui dépendent des variations locales de densité $\Delta \rho$, de compressibilité $\Delta \chi$ et d'atténuation $\Delta \alpha[1]$.

$$
\square p=\mathbf{S}(p, \Delta \rho, \Delta \chi, \Delta \alpha)
$$

Le but que se fixe l'imagerie ultrasonore est de reconstituer la carte de ces inhomogénéités avec la meilleure précision. Pour y accéder, on choisit d'illuminer une portion ou tout l'objet par un rayonnement ultrasonore connu $p_{0}(r, t)$. On observe ensuite, au niveau d'une surface de réception, le champ acoustique $p$ qui résulte de l'interaction du rayonnement incident $p_{0}$ avec l'objet. Puis, dans une dernière étape, un procédé de décodage est mis en ouvre pour dresser la carte des inhomogénéités acoustiques $(\Delta \rho, \Delta \chi, \Delta \alpha)$ à partir de la connaissance du champ $p$. Le problème qu'il faut alors résoudre est celui de l'inversion de l'équation 2. C'est un problème qu'on ne sait pas traiter dans le cas général, du fait de la relation très complexe qui existe entre $p$ et $\Delta \rho, \Delta \chi, \Delta \alpha$, et qui se manifeste par la présence de $p$ dans le terme source S. Lorsque le rayonnement incident rencontre, au sein de l'objet, des inhomogénéités de même nature, les perturbations alors engendrées se propagent à travers l'objet, tout en se modifiant différemment les unes des autres du fait des autres inhomogénéités.
Il existe cependant 2 cas où le problème peut être simplifié : d'une part si les inhomogénéités ont un effet faiblement perturbateur (cas des tissus biologiques), d'autre part si les inhomogénéités sont bien localisées et séparées par des distances suffisantes. Dans ces 2 cas on peut alors, par. un développement en petite perturbation, découpler complètement l'effet de chaque inhomogénéité sur le champ acoustique diffracté. L'ensemble des inhomogénéités de l'objet engendre un champ acoustique $p_{1}(\mathbf{r}, t)$ qui, ajouté au champ d'irradiation $p_{0}(r, t)$ (considéré comme non modifié dans l'interaction), donne la valeur de la pression observée en tout point de l'espace

$$
\begin{aligned}
& \qquad p=p_{0}+p_{1} \\
& \text { et où } \quad \square p_{0}=0 \\
& \square p_{1}=\mathbf{S}\left(p=p_{0}, \Delta \rho, \Delta \chi, \Delta \alpha\right)=S(\mathbf{r}) p_{0}(\mathbf{r}, t) .
\end{aligned}
$$

Cette approximation (Born), si elle n'est pas vérifiée dans les régions très inhomogènes contenant par exemple des structures osseuses ou des poches d'air au sein des tissus biologiques, est cependant systématiquement retenue dans les dispositifs d'imagerie médicale existant à l'heure actuelle.

- En contrôle non destructif, par contre, si les défauts ont des surfaces importantes et de faibles rayons de courbure, on ne peut plus séparer l'influence de chacun des points du défaut sur l'onde diffractée. Le fort couplage qui existe entre tous ces points se manifeste par l'apparition de différents types d'ondes parasites (creeping wave, conversion de mode, réverbération interne, etc...). Cependant, lorsque les défauts sont des cavités de petites tailles, relativement espacés les uns des autres, les ondes diffusées par chacun de ces défauts n'interagissent pratiquement pas avec les autres défauts, et les techniques de l'imagerie acoustique peuvent donner d'excellents résultats.

- L'approximation de Born, une fois justifiée, est particulièrement pratique dans la mesure où l'information acoustique utile $p_{1}(\mathbf{r}, t)$ est désormais reliée d'une façon linéaire à la pression d'irradiation $p_{0}(\mathbf{r}, t)$ et à la fonction source $S(\mathbf{r})$ qu'on cherche à déterminer (formule $3 c$ ).

- La résolution de cette équation, qui fait l'objet d'un chapitre de la théorie de la diffraction (Annexe 1), fait apparaître que chacun des points de l'objet agit comme une petite source d'onde sphérique dont l'amplitude est proportionnelle à la pression incidente $p_{0}\left(\mathbf{r}_{0}, t\right)$ et à la valeur de la fonction source $S\left(\mathbf{r}_{0}\right)$ en ce point. Le champ total $p_{1}(\mathbf{r}, t)$ s'écrit sous la forme d'une sommation de toutes ces ondes sphériques pondérées chacune par la valeur de $S\left(r_{0}\right)$. La réalisation de l'image ultrasonore de l'objet peut alors se concevoir comme un problème de reconnaissance de formes : celles des fronts d'ondes sphériques engen- 
drés pendant l'interaction, auxquels on associera la position et l'intensité des sources qui les ont engendrés.

- Sur le plan mathématique, on peut montrer qu'une fois le ćnamp acoustique $p_{1}(\mathbf{r}, t)$ enregistré au niveau d'un unique plan de détection, il existe une opération de déconvolution qui permet de retrouver l'ensemble des termes sources $S(\mathbf{r})$ connaissant l'allure du champ d'irradiation $p_{0}(\mathbf{r}, t)$ (Annexe 1). Une telle opération nécessite cependant la mise en mémoire d'une très grande quantité d'informations, à savoir un tableau à 3 dimensions dans lequel est stockée la pression $p_{1}(x, y, t), x$ et $y$ étant les coordonnées du plan de détection. Si l'on veut réaliser l'image de l'objet, en une seule illumination ultrasonore, il faut disposer d'une matrice bidimensionnelle de capteurs, de très nombreux circuits de conversion rapides $A / D(\geqslant 10 \mathrm{MHz})$, d'une mémoire extrêmement importante (plusieurs megabytes), et enfin d'un programme de déconvolution, qui bien que très simple sur le plan des principes, nécessite un temps de calcul extrêmement long. Un tel décodage numérique de l'information diffractée par l'objet est encore une opération trop lourde à effectuer. Cependant on verra plus loin qu'un certain nombre de procédés s'apparentant à cette technique de reconstruction digitale se développent actuellement.

- A côté de ces techniques de reconstitution purement digitale, il existe une très grande variété de procédés de décodage des ondes sphériques, capables de donner des images acoustiques. Le choix de la technique de décodage à utiliser dépend de nombreux facteurs.

i) La position des détecteurs relativement à la source d'illumination (imagerie en transmission ou en réflexion, mode échographique).

ii) Le codage temporel de l'illumination acoustique $p_{0}(\mathbf{r}, t)$ (signaux très brefs, signaux monochromatiques).

iii) Le codage spatial de l'illumination acoustique $p_{0}(\mathbf{r}, t)$.

On peut éclairer globalement tout l'objet en un seul tir ultrasonore. On peut utiliser une illumination plus sélective, en éclairant à chaque tir ultrasonore seulement un plan de l'objet, une ligne ou même un point de l'objet. L'accroissement de sélectivité améliore alors la qualité de l'image au détriment du temps d'acquisition des images.

- Une classification synthétique de l'ensemble des procédés d'imagerie en fonction de ces 3 facteurs est cependant loin d'être pratique. Nous lui préférons un regroupement en 6 grandes familles.

1 - Les lentilles acoustiques.

2 - L'holographie acoustique.

3 - Le traitement échographique parallèle.

4 - Le traitement échographique séquentiel.

5 - La reconstruction digitale tomographique en mode échographique.
6 - La reconstruction digitale tomographique en transmission.

1) L'approche la plus classique est celle qui utilise des lentilles acoustiques. Après une illumination globale de l'objet, le champ acoustique diffracté par l'objet est décodé au moyen de lentilles acoustiques. On peut soit travailler en transmission, soit en mode de réflexion en utilisant des sources d'irradiation situées autour de la lentille. Ces systèmes sont généralement encombrants, et les résultats obtenus en imagerie médicale et en contrôle non destructif sont décevants. En imagerie sous-marine à faible profondeur, par contre, certaines caméras ultrasonores faisant appel à des groupements de lentilles acoustiques donnent des images de très bonne qualité.

2) La deuxième approche est celle de l'holographie acoustique. On utilise ici encore une illumination globale de l'objet, mais le signal d'émission est monochromatique et le décodage des ondes sphériques peut alors être fait optiquement. On préfère cependant, après avoir enregistré l'amplitude et la phase du champ diffracté au niveau d'un plan de détection, utiliser un traitement de reconstruction digitale. Les résultats obtenus en imagerie médicale sont cependant très médiocres. En contrôle non destructif, par contre, des images correctes ont pu être obtenues. En acoustique sous-marine, des dispositifs holographiques fonctionnant en mode de réflexion ont été réalisés, qui donnent pour certains des images remarquables.

3) La troisième approche est celle du traitement échographique "en parallèle ". On illumine en un seul tir ultrasonore par un signal bref, une tranche de l'objet à étudier. Au cours de la réception des échos à partir d'une barrette multiple de transducteurs, on traite en parallèle de façon analogique tous ces signaux, afin de reconstituer en temps réel l'image de la coupe échographique. Ces techniques permettent d'obtenir une cadence d'image très élevée $(1$ image par tir). Différents procédés ont été proposés en imagerie médicale, la qualité des images est cependant mauvaise. En imagerie sous-marine, les techniques dites de "Beam Forming" sont arrivées à un stade de raffinement supérieur et donnent de bons résultats.

4) La quatrième approche, la plus connue, est celle de l'échographie ultrasonore séquentielle (échographie en mode $B$ et en mode C). On utilise des signaux brefs, et on remplace l'illumination globale de l'objet par une illumination sélective, en éclairant à chaque tir une zone relativement restreinte de l'objet. Des dispositifs de focalisation doivent être mis en œuvre pendant la réception des échos, et l'image est formée séquentiellement au cours du temps en déplaçant la ligne de tir. Ce sont ces techniques qui donnent à l'heure actuelle les meilleurs résultats en imagerie médicale, sous la forme de coupes échographiques présentées en mode B. En contrôle non destructif, 
comme en acoustique sous-marine, ces dispositifs donnent de bons résultats.

5) La cinquième approche, plus récente, est celle de la reconstruction tomographique en mode échographique. On parle aussi souvent d'ouverture synthétique à focalisation digitale. L'illumination d'une tranche de l'objet se fait séquentiellement, soit au moyen d'ondes sphériques dont l'origine est déplacée à chaque tir, soit au moyen d'ondes planes dont l'incidence relativement à l'objet change au cours du temps. On enregistre à chaque tir le champ diffracté en mode échographique au moyen d'un ou de plusieurs transducteurs (barrette linéaire, barrette cylindrique, matrice de transducteurs). Un traitement de reconstruction digitale, faisant appel à des algorithmes de projection inverse, permet de réaliser l'image finale. De très bons résultats ont été obtenus par cette technique en contrôle non destructif en travaillant à des fréquences qui peuvent atteindre $50 \mathrm{MHz}$. Les premiers résultats en contrôle médical semblent très prometteurs.

6) La dernière approche est celle de la tomographie en transmission. Ces techniques ont été dans un premier temps calquées sur la tomographie par rayons X. Elles nécessitent d'effectuer un nombre de tirs ultrasonores égal au nombre de points de l'image qu'on cherche à reconstituer. Ce sont donc des techniques lentes. On peut obtenir par des procédés de reconstruction numérique soit une cartographie de l'atténuation ultrasonore à une fréquence, soit par la technique dite du temps de vol une cartographie de la célérité ultrasonore. Les résultats en imagerie médicale sont cependant encore décevants et restent limités par l'utilisation de l'imagerie en mode de transmission, qui ne s'applique correctement que dans l'examen du sein. En contrôle non destructif, la cartographie des vitesses ultrasonores semble intéressante car, dans certains cas, elle peut être reliée à la cartographie des contraintes résiduelles à l'intérieur du matériau.

\section{Les techniques de l'imagerie ultrasonore.}

2.1 Les LeNTILles ACOUSTiQUes. - Le procédé de décodage le plus classique utilise des lentilles acoustiques. On sait qu'une telle lentille est capable d'effectuer en temps réel l'opération de décodage souhaitée. Elle se comporte en effet comme un dispositif retardateur capable de transformer chacun des fronts d'ondes sphériques divergents en provenance de l'objet en un front d'onde convergent suivant un stigmatisme rigoureux. On forme ainsi d'une façon naturelle l'image de l'objet, plan par plan, sans faire appel à aucun traitement digital. L'opération de décodage se fait en parallèle sur toute l'ouverture de la lentille de façon quasi instantanée. Les lentilles ultrasonores sont généralement réalisées à partir de milieux solides (plexiglas, polystyrène), dans lesquels la vitesse de propagation des ultrasons est supérieure à celle de l'eau (la concavité de ces lentilles est inverse de celle des lentilles optiques). La réalisation de lentilles de bonne qualité pose un certain nombre de problèmes car il fảut limiter les réflexions parasites de l'interface liquide-solide, ainsi que les conversions d'ondes longitudinales incidentes en ondes transversales dans ces lentilles solides. Un usinage précis des lentilles permet de corriger les aberrations de sphéricité et de coma. L'association de plusieurs lentilles corrige les aberrations chromatiques [2]. Des écrans permettant d'apodiser la tache focale peuvent être mis en œuvre, certains ont été réalisés en polyuréthane, dont l'impédance acoustique est adaptée à celle de l'eau, et qui présente un coefficient d'absorption important, ce qui permet d'obtenir facilement une modulation d'amplitude de type gaussienne, en modulant l'épaisseur de l'écran suivant une loi quadratique.

Indépendamment des problèmes posés par la réalisation des lentilles, les images ultrasonores ainsi obtenues doivent être perçues par une rétine ultrasonore. L'électronique associée peut être relativement simple, car la mesure en chaque point de l'intensité acoustique moyenne suffit pour réaliser l'image. Dès 1929 , le physicien russe Sokolov proposa une première rétine ultrasonore constituée d'un tube d'oscilloscope dont la face fluorescente est remplacée par une lame piézoélectrique (Fig. 2). L'image ultrasonore est convertie en une cartographie de potentiel électrique qui apparaît sur la face interne de la lame, et qui peut être

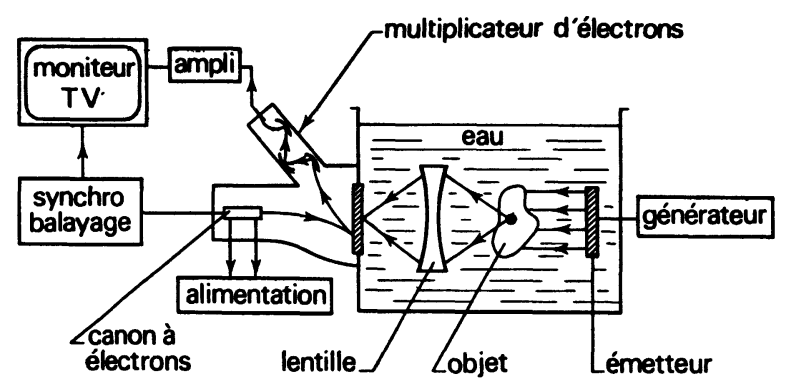

Fig. 2. - Principe du tube de Sokoloff.

[The Sokoloff tube.]

lue rapidement par un faisceau électronique balayé comme le spot d'un récepteur de télévision. La société EMI a réalisé des caméras de ce type très performantes [3] qui, associées à des lentilles de polystyrène, permettent d'obtenir en mode de réflexion des images sous-marines de bonne qualité jusqu'à $30 \mathrm{~m}$ en fonctionnant à une fréquence de $2 \mathrm{MHz}$, avec une résolution angulaire de $1^{\circ}$ sur un champ de $40^{\circ}$ (Fig. 3). D'autres dispositifs faisant appel à une barrette linéaire de détecteurs ont été développés. Dans le cas de la caméra de Green, développée pour l'application médicale [4], (Fig. 4) fonctionnant à $3 \mathbf{M H z}$, on utilise une barrette de 192 transducteurs. L'image de chaque ligne d'un plan de l'objet est déplacée au niveau de la barrette de réception en utilisant un système de prismes 


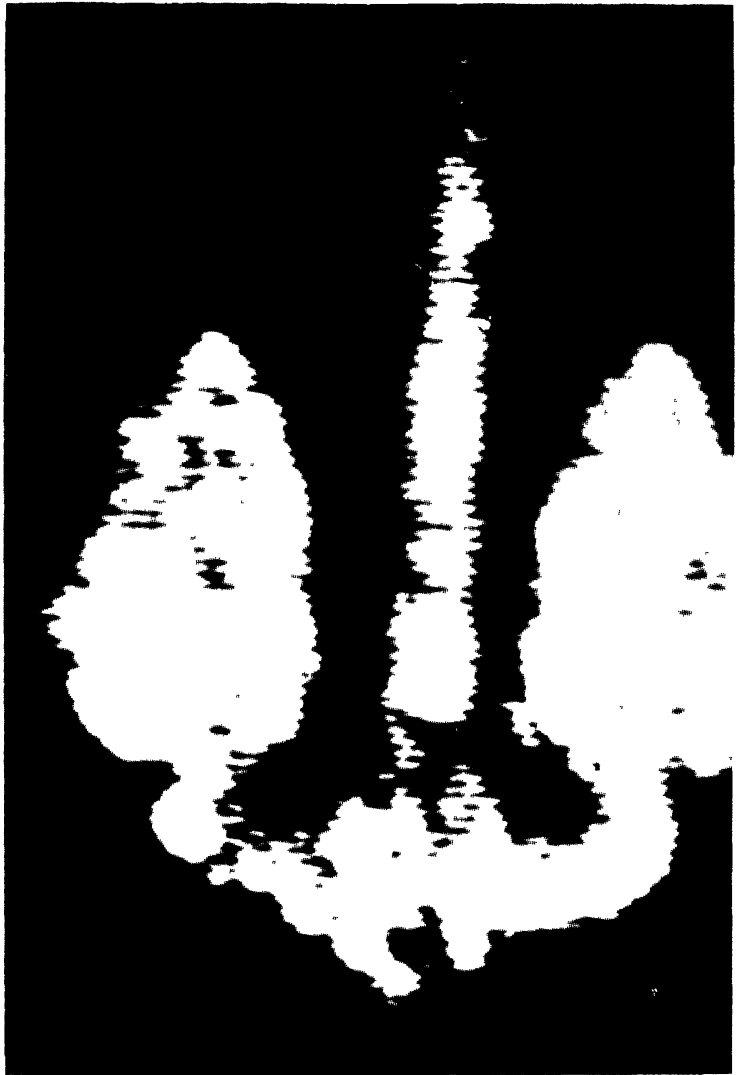

Fig. 3. - Image d'une ancre obtenue avec la caméra E.M.I.

[Ultrasonic image of anchor obtained from the EMI camera.]

acoustiques tournants. La rotation mécanique des prismes permet l'obtention de 8 à 10 images par seconde sans créer de turbulence. Les résultats obtenus en transmission, comme en réflexion, par ce dispositif dans le domaine médical sont cependant décevants.
D'autres dispositifs faisant appel à des barrettes de transducteurs ont été développés en imagerie sousmarine. $H$. Warner et $D$. Folds ont mis au point au Naval Coastal Systems Center, en Floride [5], une caméra d'un mètre de diamètre formée de 6 éléments régulés en température où l'image acoustique est lue au moyen d'une barrette de transducteurs en rotation rapide. Le système fonctionne à $870 \mathrm{kHz}$, il utilise 4 émetteurs ultrasonores montés autour des lentilles, et permet d'obtenir une résolution de 0,12 degré jusqu'à une profondeur de $100 \mathrm{~m}$ (Fig. 5).

Un autre dispositif du même type a été mis au point en France par le GESMA [6]. Une barrette de 64 transducteurs analyse l'image acoustique provenant des lentilles à travers un miroir tournant. Ce système fonctionne autour de $2 \mathrm{MHz}$, la technologie des transducteurs permet d'atteindre une grande ouverture angulaire (Fig. 6).

Il faut remarquer que tous ces dispositifs sont nécessairement encombrants, du fait de la distance qui doit exister entre le plan détecteur et la lentille. Ce n'est pas un obstacle majeur en acoustique sousmarine. Par contre, en acoustique médicale, comparée aux faibles encombrements de certains dispositifs échographiques, c'est une limitation importante qui explique qu'après quelques tentatives comme celle de Green de tels projets ont été abandonnés.

2.2 L'HOLOGRAPHIE ACOUSTIQUE. - Parallèlement à ces études sur les lentilles acoustiques, les premiers succès de l'holographie optique au début des années 60 ont orienté une partie de la recherche en imagerie ultrasonore vers les techniques de décodage holographique. On sait qu'en holographie, le choix d'une illumination de l'objet par un signal monochromatique cohérent permet d'enregistrer simplement les caracté-

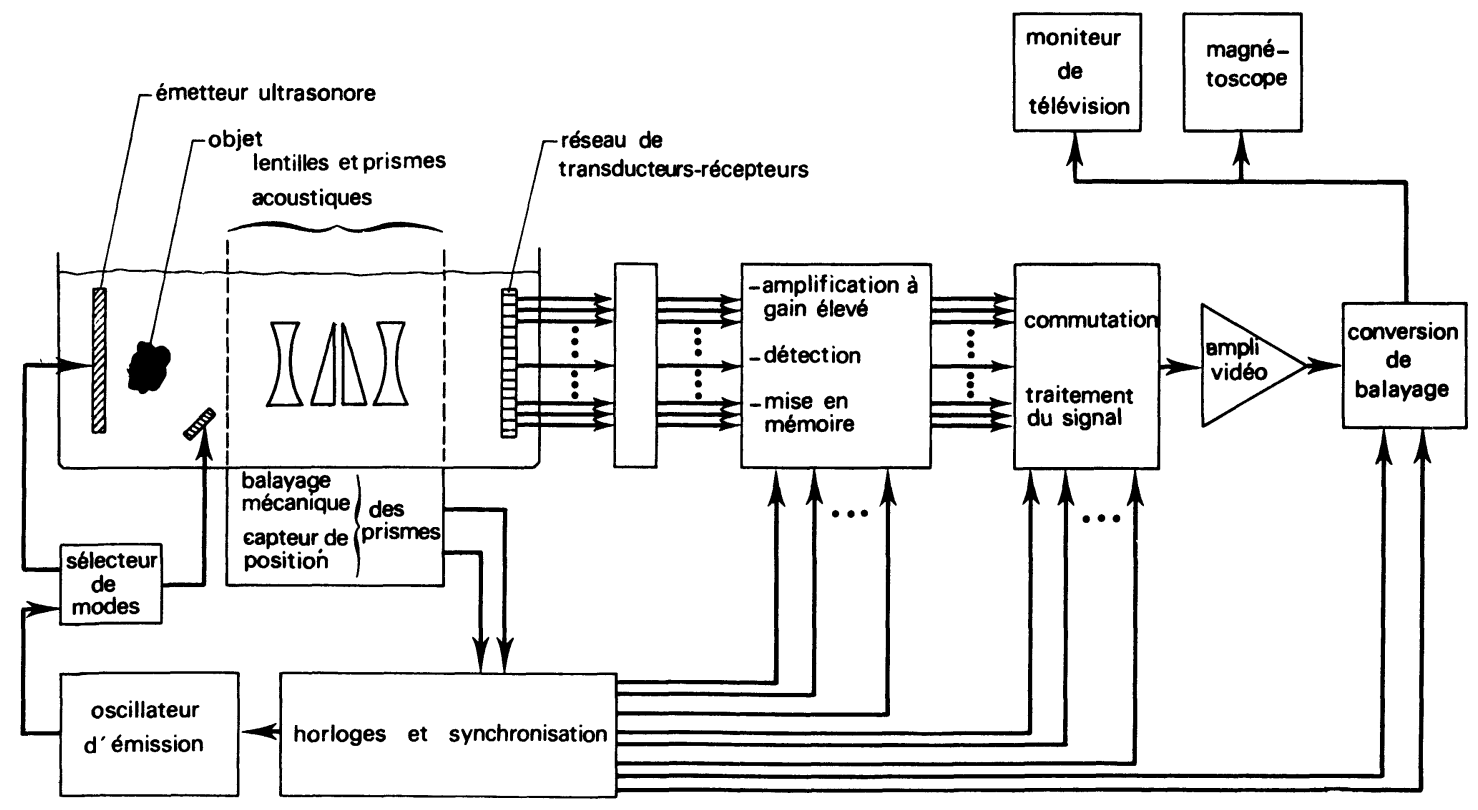

Fig. 4. - Caméra ultrasonore de Green. La rétine ultrasonore est une barrette de 192 éléments.

[Green camera using a 1.92 transducers array.] 


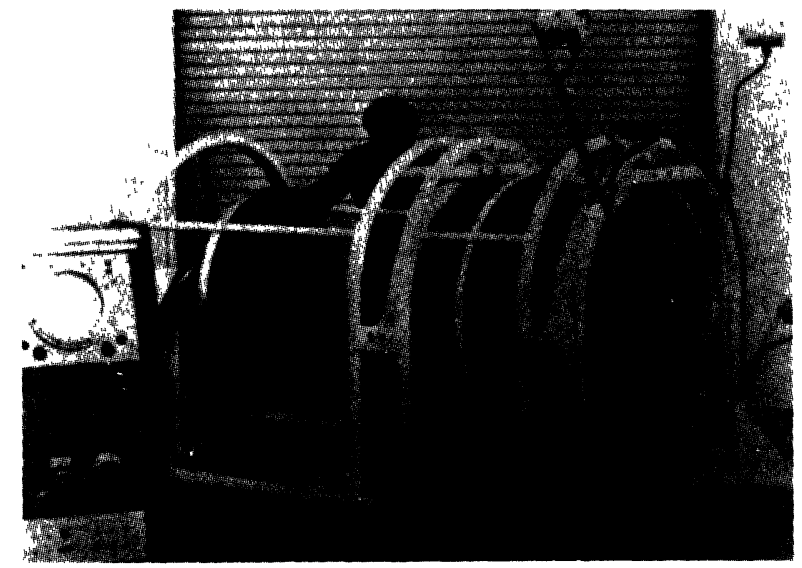

Fig. 5. - Caméra ultrasonore sous-marine [5]. Elle est formée de 6 lentilles et d'une barrette linéaire rotative. On peut distinguer les 4 émetteurs ultrasonores.

[Submarine ultrasonic camera [5] (6 lenses, 4 transmitters).]

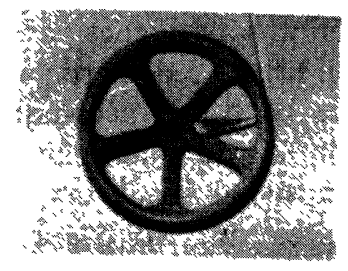

a) Optic inage

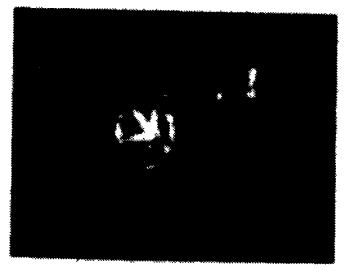

c) Distance $3 \mathrm{~m}$ $45^{\circ}$ incidence

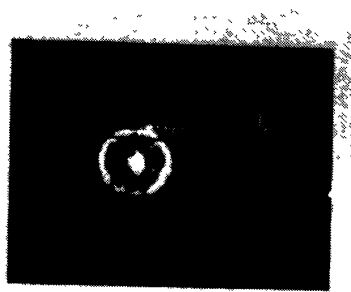

b) Distance $3 \mathrm{~m}$ Normal incidence

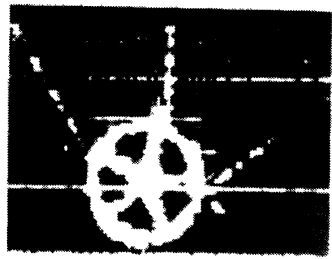

d) Distance $2.2 \mathrm{~m}$ normal incidence

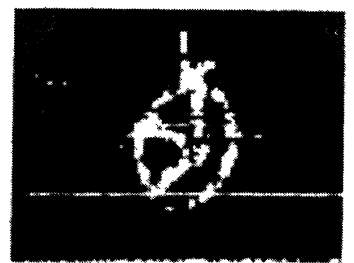

e) Distance $2,45^{\circ}$ incidence

Fig. 6. - Résultats obtenus par la caméra du G.E.S.M.A. sur une roue de $20 \mathrm{~cm}$ de diamètre.

[Ultrasonic images obtained from the GESMA camera.]

ristiques de l'onde diffractée par l'objet, au niveau d'un plan de détection, en la faisant interférer avec une onde de référence 7. A partir de cette photographie instantanée de l'onde diffractée (somme de toutes les ondelettes sphériques élémentaires diffractées), on cherche à remonter à une image de l'objet en effectuant une opération de décodage. Cette opération de déco- dage, qui consiste à reconnaître la signature de chacune des ondelettes sphériques élémentaires, peut être effectuée de façon simple en illuminant la transparence holographique par une onde cohérente identique à l'onde de référence. La diffraction réalise alors d'une façon naturelle l'opération de déconvolution nécessaire à la reconstitution de l'objet (Annexe 2).

En acoustique, la réalisation d'une illumination cohérente monochromatique ne pose pas de problème. Une première approche [8] utilise comme plan de détection l'interface liquide-air. Sous l'action de la pression de radiation ultrasonore, la surface subit une déformation qui suit les variations de l'intensité acoustique (Fig. 7). L'hologramme acoustique qu'on

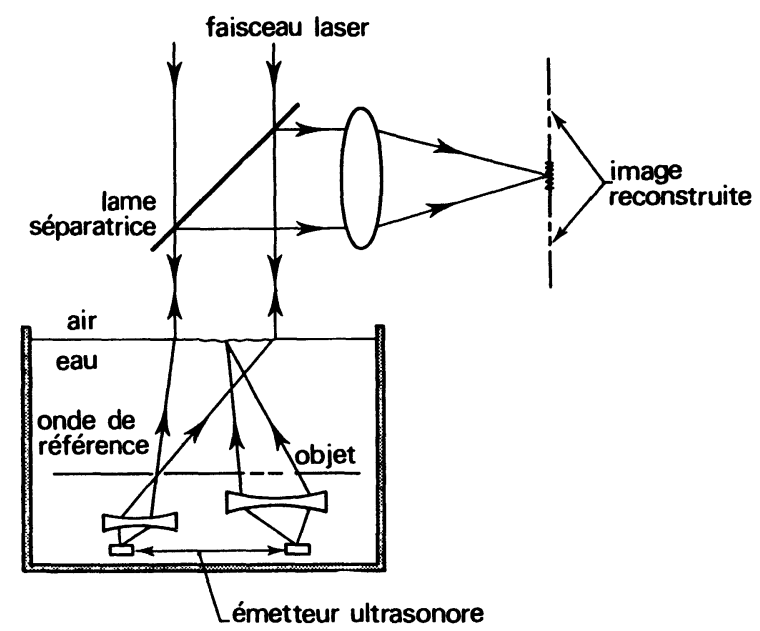

Fig. 7. - Schéma de la technique d'holographie acoustique par relief de surface. Le plan de détection est l'interface liquide-air. Sous la pression de radiation ultrasonore, la surface subit une déformation. L'hologramme acoustique obtenu peut être décodé optiquement par le faisceau laser.

[Optical holographic restitution of an acoustical hologram obtained at the liquid-gas interface.]

peut alors réaliser en faisant interférer une onde acoustique de référence avec l'onde diffractée par l'objet, peut alors être décodé optiquement par un faisceau laser. La diffraction optique de cette onde sur la surface déformée réalise la transformée de Fresnel souhaitée pour reconstituer l'image de l'objet. Ce procédé de décodage optique souffre cependant de nombreuses aberrations dont la plus importante provient de la grande différence entre les longueurs $\mathrm{d}^{3}$ onde acoustiques et optiques qui conduit à une déformation importante de l'image reconstruite. Il est d'autre part peu sensible et sa dynamique est faible. Une autre approche en holographie acoustique consiste à réaliser des surfaces matricielles de récepteurs ultrasonores dont on peut recueillir séquentiellement l'information. On bénéficie alors du fait que les transducteurs ultrasonores sont linéaires et peuvent donner une information de phase et d'amplitude, en leur associant une électronique appropriée (détection synchrone). Cette information peut alors être fournie à 
un ordinateur qui effectuera le calcul rigoureux correspondant à la reconstitution, ce qui permettra d'éviter les aberrations précédemment décrites. Une matrice de $256 \times 256$ transducteurs électrostatiques au pas de $1 \mathrm{~mm} \times 1 \mathrm{~mm}$ a été réalisée par $P$. Alais [9]. Une commutation électronique rapide des transducteurs permet d'interroger toute la surface en $1 / 16$ de seconde et donc d'obtenir des hologrammes en temps réel (Fig. 8). Plus récemment, différentes équipes ont

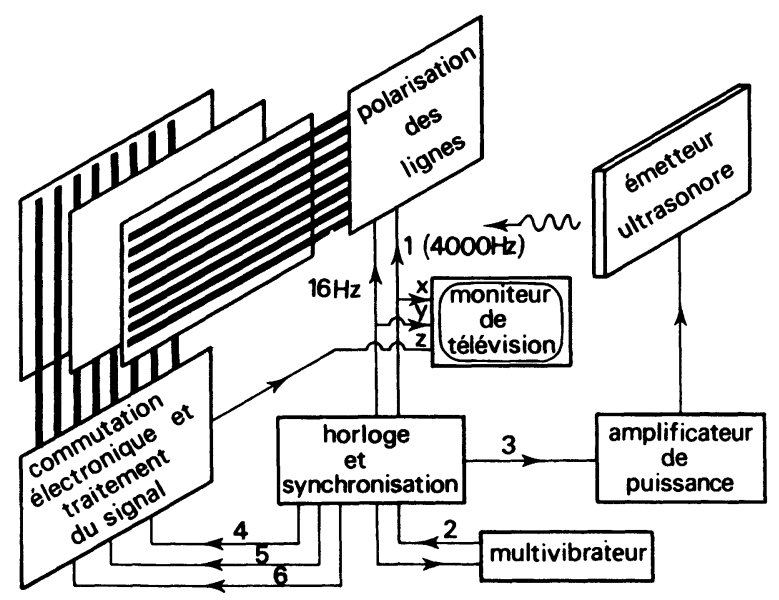

Fig. 8. - Holographie acoustique avec une rétine phonosensible électrostatique. Méthode mise au point par P. Alais. La rétine est constituée de transducteurs capacitifs localisés aux croisements des électrodes lignes et colonnes de deux circuits imprimés enserrant une feuille diélectrique $(256 \times 256$ au pas de $1 \mathrm{~mm}$ ). Les hologrammes sont obtenus en temps réel.

[Acoustical holography with a $2 \mathrm{D}$ electrostatic transducer array.]

mis au point des matrices de transducteurs piézoélectriques de grande sensibilité, où chaque transducteur est équipé d'une électronique d'amplification et de détection synchrone qui permet d'enregistrer l'information de phase et d'amplitude [10-12]. De tels sy'stèmes peuvent fonctionner en transmission ou en réflexion, en utilisant alors une illumination quasimonochromatique provenant du même plan d'émission. Un système tout à fait remarquable a été mis au point par la société japonaise OKI Electronic Corporation [13], qui fait appel d'une part à une matrice $4 \times 4$ émetteurs (Fig. 9) et d'autre part à une matrice de réception de $32 \times 32$ récepteurs. L'image finale est obtenue en effectuant 64 tirs ultrasonores à des incidences différentes (en jouant sur les phases des signaux délivrés aux émetteurs). A chaque tir on obtient, par une mesure de phase et d'amplitude, un hologramme sur les $32 \times 32$ récepteurs. Cet hologramme est décodé digitalement en réalisant la transformée de Fresnel inverse et l'on obtient une image de $32 \times 32$ points. L'image finale est obtenue en imbriquant correctement les 64 images élémentaires. On obtient ainsi une image de $256 \times 256$ points. Ce système fonctionne à $200 \mathrm{kHz}$, la résolution obtenue est de l'ordre de $0,4^{\circ}$, la profondeur d'exploration est comprise entre 2 et $100 \mathrm{~m}$. Les images obtenues à partir de ce dispositif sont remarquables (Fig. 9). Des systèmes du même type sont développés dans le domaine de l'acoustique sous-marine par Bendix (250 kHz) et par l'équipe du Naval Oceans Systems Center à San Diego $(642 \mathrm{kHz})[14,15]$.

Dans le domaine du contrôle non destructif, un certain nombre de systèmes holographiques ont été mis au point. Ils font appel généralement à une seule barrette linéaire de transducteurs, qui peut être déplacée mécaniquement dans la direction orthogonale au réseau de transducteurs $[16,17]$. Le temps d'obtention des hologrammes n'est pas aussi critique ici qu'en acoustique sous-marine ou qu'en imagerie médicale, du fait de l'absence de mouvements des objets examinés.

Un des systèmes les plus efficaces qui ait été réalisé combine une source d'illumination focalisant sur une ligne (grand transducteur cylindrique) à une barrette de réception de 120 éléments opérant à $2,3 \mathrm{MHz}$. La source et le réseau de réception sont alignés parallèlement les uns aux autres (Fig. 10). La reconstruction holographique se fait après le balayage mécanique de la barrette et elle donne, du fait de l'illumination sélective, un très bon rapport signal sur bruit. En inclinant plus ou moins le faisceau ultrasonore incident, on peut travailler soit en mode longitudinal, soit en mode transverse pour examiner des défauts inclinés (Fig. 11).

Dans le domaine des applications médicales de l'holographie acoustique, les résultats sont décevants, et ceci malgré l'utilisation de procédés de décodage holographique apparemment idéaux (grande ouverture, échantillonnage suffisant; grande dynamique). Cette constatation peut en fait s'expliquer en remarquant qu'en holographie ultrasonore médicale, l'illumination globale de l'objet entraîne que la reconnaissance de chacun des points sources se fait en présence d'un bruit important, provenant de l'ensemble des autres points de l'objet. Ce bruit d'interférence (analogue au speckle optique) réduit en pratique le pouvoir de résolution de façon importante surtout lorsque l'objet à étudier contient de nombreuses inhomogénéités. En outre, en holographie, l'utilisation comme source d'illumination d'un signal monochromatique, qui ne peut localement fournir que deux informations (amplitude et phase) et ceci quelle que soit la complexité de l'objet étudié, n'est certainement pas un bon choix pour l'étude d'organes de structure complexe. Le décodage des coordonnées de chacune des sources, en présence de bruit, se fait avec d'autant plus d'efficacité que la signature de chaque source sur le plan de détection contient beaucoup d'informations.

En augmentant la dimension des ouvertures détectrices, on peut espérer accéder à une quantité d'informations plus élevée. Cependant, en acoustique, les difficultés rencontrées pour réaliser des rétines ultra- 

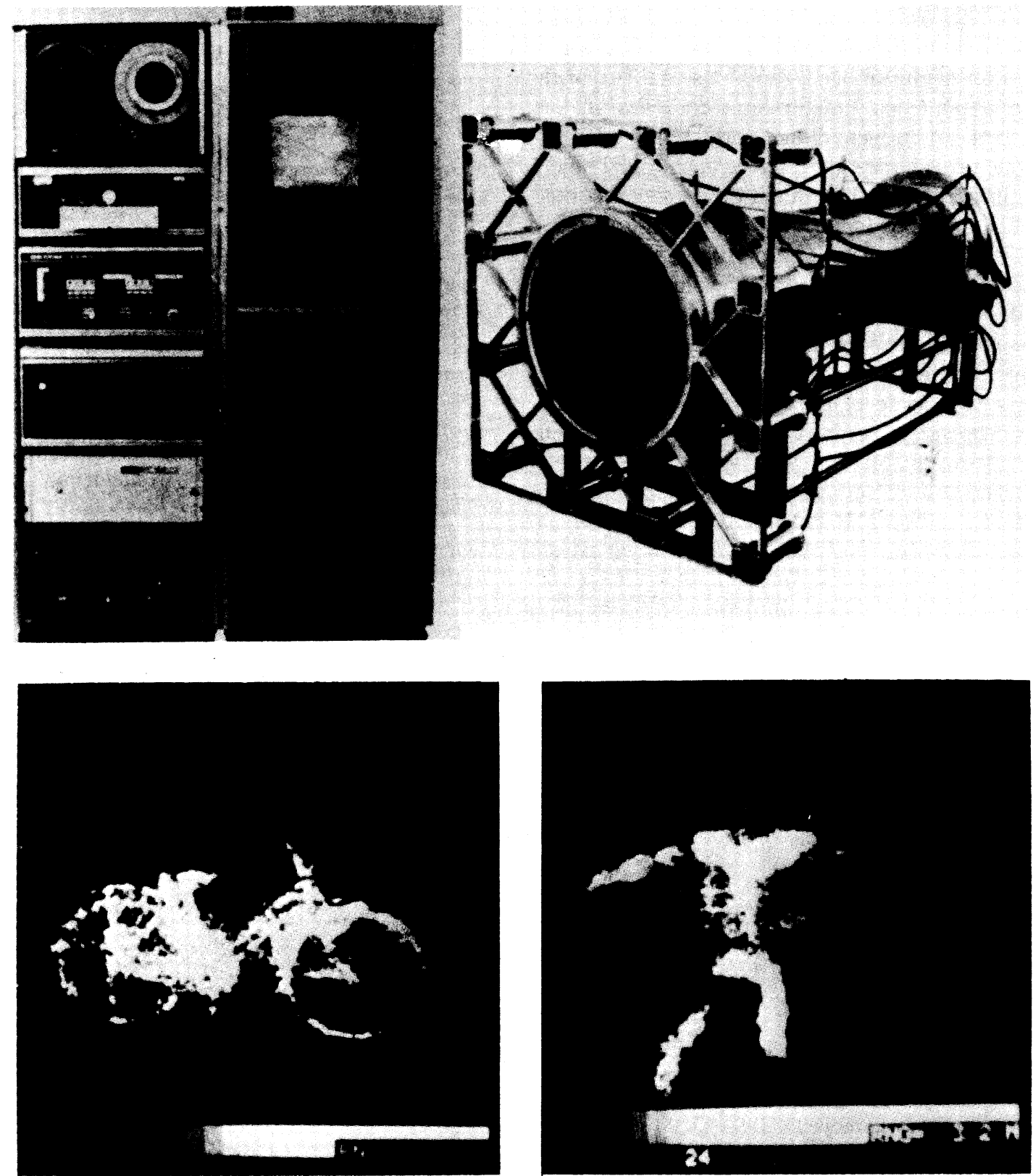

Fig. 9. - En haut : dispositif d'holographie acoustique marine développé par O.K.I. Il est composé de $32 \times 32$ récepteurs et de $4 \times 4$ émetteurs. En bas : reconstruction de l'image d'une bicyclette et d'un plongeur situés à 3,2 $\mathrm{m}$ de l'ouverture.

[Submarine acoustical holography from O.K.I. (above). Reconstructed images from a bicycle and from a diver located at a depth of 3.2 meters (below).]

sonores de grande taille limitent en fait les ouvertures utilisées à des dimensions de l'ordre de quelques centaines de longueurs d'onde. Dans les meilleurs cas, les $2 N^{2}$ informations acquises en holographie acoustique par une rétine matricielle échantillonnée par $N^{2}$ transducteurs (où $N$ reste jusqu'à présent inférieur à 300) sont insuffisantes pour reconstituer avec précision les trois dimensions d'un objet biologique de dimensions raisonnables.

Il faut noter ici que ce problème ne se pose pas avec la même acuité en optique, où les ouvertures utilisées contiennent un nombre beaucoup plus important de longueurs d'onde $\left(\approx 5 \times 10^{4} \times 5 \times 10^{4} \lambda^{2}\right)$ ce qui permet d'obtenir une information beaucoup plus riche sur des objets qui en outre, il faut le souligner, ne sont pas de véritables objets tridimensionnels. En optique, dans la plupart des cas, seule la surface des objets diffuse la lumière, et l'information qu'elle délivre et qu'on cherche à décoder n'a en fait qu'un caractère bidimensionnel alors qu'en acoustique médicale, la relative transparence des organes au rayonnement ultrasonore de quelques $\mathrm{MHz}$ en font de véritables objets tridimensionnels.

En contrôle non destructif et en imagerie sousmarine, par contre, la complexité des objets à l'échelle de la longueur d'onde est beaucoup moins grande, 
c'est ce qui explique la meilleure efficacité des techniques holographiques dans ces domaines. Une amélioration non négligeable du rapport signal sur bruit peut être obtenue en fonctionnant en réflexion, en sélectionnant au moyen d'une porte temporelle les signaux ne provenant que d'une tranche de l'objet, à condition d'utiliser à l'illumination un signal contenant un nombre de sinusoïdes suffisant (quelques dizaines) pour lequel la notion de phase a encore un sens. On peut ainsi améliorer la résolution en profondeur des techniques holographiques, qui est généralement mauvaise, tout en diminuant le bruit de fond acoustique. De telles techniques sont exploitées en imagerie sous-marine et en contrôle non destructif. Elles n'ont cependant pas été beaucoup développées en imagerie médicale, où l'on s'est vite rendu compte que l'utilisation d'un codage temporel beaucoup plus riche devait s'imposer. Il est apparu que la façon la plus simple d'enrichir l'information acoustique est de
GEOMETRIES FOR HIGH-SPEED IMAGING

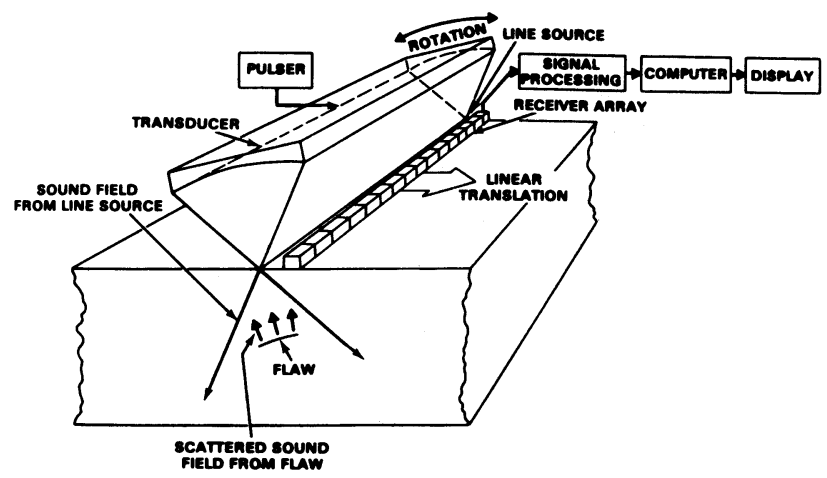

Fig. 10. - Schéma de principe d'un système combinant une focalisation sur une ligne à une barrette de réception posée sur le matériau à étudier.

[Geometry of a 1D holographical device.]

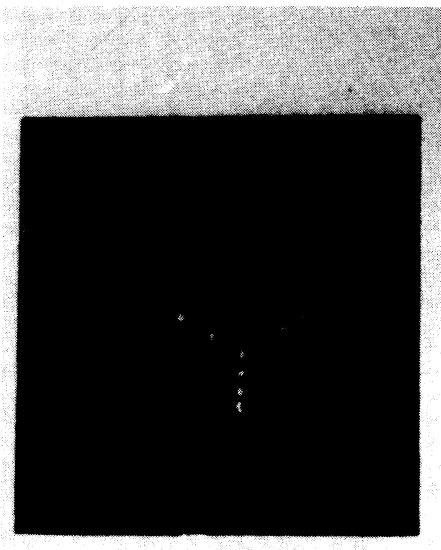

(a)

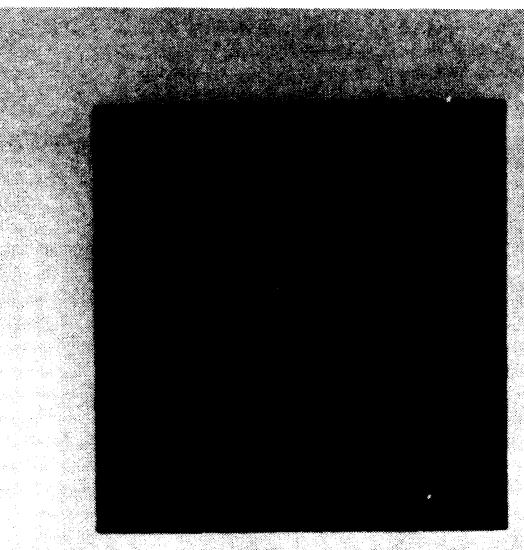

(b)

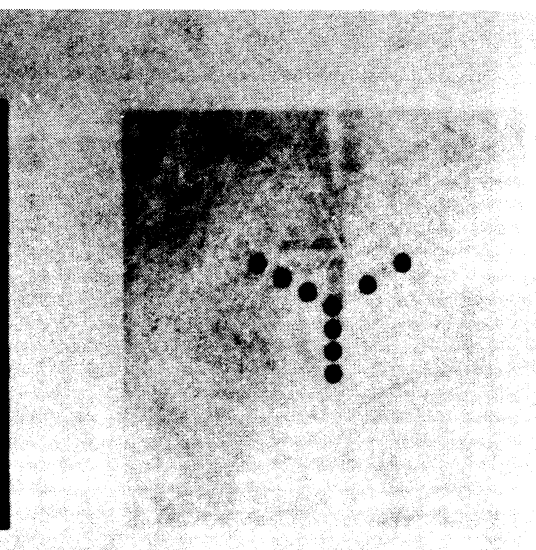

(c)

Fig. 11. - Images de défauts obtenus en mode de cisaillement.

[Defects imaging in shear mode.]

revenir à des signaux d'illumination brefs, tout en travaillant en échographie.

2.3 TRAITEMENT ÉCHOGRAPHIQUE PARALlèle. - Travailler en mode échographique consiste à choisir pour plan de réception un plan identique au plan d'émission tout en illuminant l'objet par un signal très bref. On bénéficie alors d'une information supplémentaire pour localiser les inhomogénéités de l'objet, en tenant compte du temps d'arrivée de chacun des fronts d'onde diffusés par ces inhomogénéités. Pour un signal d'illumination bref, on peut alors reconnaître chacune des cibles à travers le rayon et le centre de courbure du front d'onde réfléchi, mais aussi en tenant compte du temps d'arrivée du début de ce front d'onde au niveau du plan de réception $(\tau=2 \mathrm{z} / \mathrm{c}$ où $z$ est la distance de la cible au plan d'émissionréception), temps qui est lié de façon univoque à la profondeur de la cible. Cette information est à rapprocher de celle obtenue en imagerie par transmission, où tous les fronts d'onde engendrés dans l'objet par le faisceau incident, arrivent simultanément au niveau du plan de réception. C'est une information moins riche qu'en échographie, qui ne donne la valeur de la coordonnée $z$ des cibles, qu'à travers la courbure du front d'onde. Les signaux provenant des différentes inhomogénéités de l'objet arrivent tous ensemble sur le plan de réception, en introduisant un bruit de speckle très défavorable à l'opération de décodage.

En échographie, par contre, le décodage naturel de la coordonnée $z$ entraîne une importante diminution de ce bruit, qui ne provient plus que d'une tranche de l'objet d'autant plus fine que le signal d'illumination est bref. En tenant compte des signaux d'illumination réalisables à partir de transducteurs bien amortis, on peut atteindre des durées d'illumination de l'ordre de la microseconde, ce qui, comparé aux $200 \mu$ s nécessaires pour explorer une profondeur de $15 \mathrm{~cm}$, donne une idée de la quantité d'information qu'on peut obtenir. La richesse de cette information 
est telle que les premiers systèmes échographiques qui n'exploitaient pratiquement que cette donnée temporelle ont fourni de bonnes images.

On doit insister ici sur le fait que la possibilité d'utiliser l'information échographique pour visualiser des structures de petite dimension $(\sim \mathrm{mm})$ est spécifique à l'acoustique. C'est la très faible célérité ultrasonore, en regard de la vitesse de propagation des ondes électromagnétiques, qui permet de séparer sans difficulté les échos provenant des cibles très proches.

Cependant, le choix d'un mode d'exploration échographique, s'il semble le plus favorable, pose en acoustique un certain nombre de problèmes. Le problème fondamental est bien entendu lié à l'opération de décodage qu'on doit effectuer pour reconstituer avec précision la cartographie des inhomogénéités. Si l'on veut bénéficier pleinement des avantages liés au décodage naturel de la coordonnée $z$ qu'apporte l'échographie, il faut développer des procédés de décodage en temps réel, capable au fur et à mesure du retour des échos d'effectuer une opération de déconvolution qui soit adaptée à chaque instant à la courbure des fronts d'ondes observés. Un tel procédé de décodage peut en fait se concevoir comme un véritable «zoom» que réaliserait par exemple une lentille acoustique à focale très rapidement variable, qui serait placée sur le trajet du faisceau acoustique réfléchi. On observerait alors au niveau d'un unique plan de détection la séquence des images de chacun des plans de l'objet illuminé par le faisceau acoustique d'exploration, la variation rapide de focale $(\Delta f / \Delta t \simeq$ $3 \mathrm{~mm} / \mu$ s dans l'eau) permettant à chaque instant de mettre au point sur l'image du plan dont les échos arrivent sur le plan de détection.

Si une telle opération de décodage est parfaitement utopique en utilisant des lentilles acoustiques de type classique, on peut cependant, dans le cas où l'on restreint l'exploration de l'objet à celle d'un unique plan de coupe, envisager de remplacer l'effet retardateur d'une telle lentille par l'action d'un ensemble de lignes à retard connectées à une barrette de transducteurs qui joueraient le rôle de face d'entrée de cette lentille. L'ensemble des signaux électriques retardés excite alors une deuxième barrette de transducteurs qui, faisant office de face de sortie de la lentille, délivrerait dans un deuxième milieu de propagation un rayonnement acoustique qui, à travers l'effet de la propagation, réaliserait l'image acoustique du plan qu'on cherche à observer (Fig. 12).

La focale instantanée d'un tel système dépend d'une part de la distance $z^{\prime}$ à laquelle on veut restituer l'image de chaque ligne de l'objet, et d'autre part de la profondeur $z=c t / 2$ des cibles observées au temps $t$.

$$
\frac{1}{f}=\frac{1}{z}-\frac{1}{z^{\prime}}=\frac{2 z^{\prime}-c t}{c t z^{\prime}}
$$

La correction quadratique de retard qui doit être appliquée par les lignes à retard électroniques pour

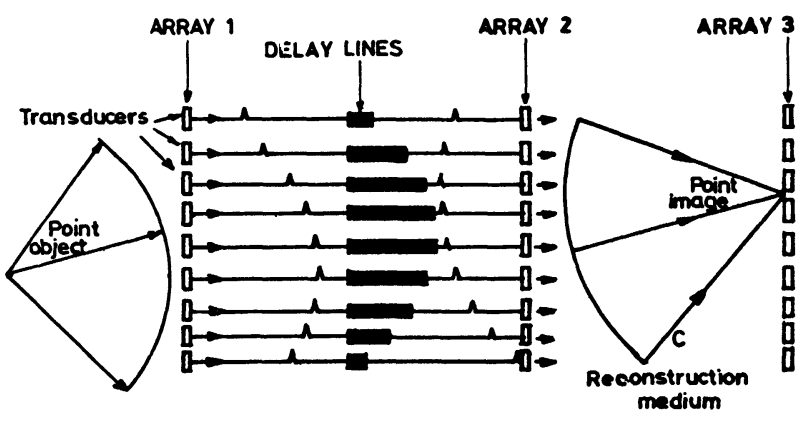

Fig. 12. - Lentille électronique obtenue à partir de 2 réseaux linéaires de transducteurs et d'un groupe de lignes à retard. La $3^{\mathrm{e}}$ barrette est utilisée pour observer l'image acoustique. Chaque onde sphérique divergente est transformée en une onde sphérique convergente.

[Electronic lens with delay lines and 2 transducers arrays, the third array is used to observe the acoustical image.]

simuler cette focale va dépendre du temps suivant :

$$
\Delta \tau\left(x_{\mathrm{n}}, t\right)=\frac{-x_{\mathrm{n}}^{2}}{2 c f}=\frac{-x_{\mathrm{n}}^{2}}{c^{2}}\left(\frac{1}{t}\right)+\frac{x_{\mathrm{n}}^{2}}{2 c z^{\prime}}
$$

$x_{\mathrm{n}}$ étant la coordonnée de chacun des transducteurs de la barrette.

Une telle solution, qui permettrait d'accéder à la structure bidimensionnelle d'une coupe échographique au cours d'un seul tir ultrasonore, si elle est séduisante, pose en fait le problème de l'utilisation de lignes à retard continûment variables qui, pour les lignes de type $\mathrm{CCD}$, n'atteignent pas à ce jour la dynamique requise en échographie ( $>80 \mathrm{db}$ ). En outre, les aberrations de sphéricité de ce type de lentilles limitent sévèrement le champ d'exploration à des points situés près de l'axe du système.

Une autre technique, faisant toujours appel à trois barrettes de transducteurs, peut être envisagée, qui permettrait de s'affranchir de toutes les aberrations. Elle nécessite cependant le remplacement des lignes à retard variables par des circuits de mémorisation électronique. Ces circuits enregistreraient, dans un premier temps, les signaux échographiques provenant de l'objet et, dans un deuxième temps, réinjecteraient ces signaux sur la deuxième barrette, tout en inversant systématiquement l'ordre d'arrivée, les premières informations recueillies étant les dernières informations à sortir. On peut alors réaliser ainsi un véritable retournement du temps (en temps différé (Annexe 1)). Une onde sphérique divergente, qui aurait été enregistrée au niveau de la première barrette, se transforme alors, par retournement du temps, en une onde sphérique convergente qui, réémise dans le deuxième milieu de propagation, donnera un point image du point objet, sans aucune des aberrations de sphéricité présentées par les lentilles. Un tel concept s'apparente à celui de la reconstitution holographique où, après l'enregistrement de la phase du champ diffracté, une inversion de cette phase, suivie d'une opération de propagation directe (transformée de Fresnel), permet la 
reconstitution de l'objet. C'est cependant une technique beaucoup plus générale qu'en holographie monochromatique, dans la mesure où ce procédé de décodage fonctionne pour n'importe quel type de signal d'illumination. On peut ainsi exploiter les possibilités de l'échographie en utilisant des signaux très brefs.

Jusqu'à présent, cependant, les deux techniques que nous avons décrites n'ont pas été réalisées sous cette forme. Elles ont cependant été mises en œuvre dans le cas de signaux d'illumination d'allure sinusoïdale suffisamment long pour qu'on puisse parler de leur phase. Dans ce cas, la correction quadratique de retard envisagée dans la première méthode peut être remplacée par une correction quadratique de phase.

$$
\Delta \phi\left(x_{n}\right)=\omega \Delta \tau\left(x_{n}\right)=-\frac{\pi}{\lambda f} x_{n}^{2} .
$$

Un premier système a été proposé par Torguet [18], faisant appel à une technique de décodage optique où, après illumination d'un plan de tir ultrasonore, l'information échographique recueillie par la barrette de transducteurs est transformée au moyen d'un convertisseur acousto-optique, sous forme d'une modulation de phase spatiale d'un faisceau optique. Le zoom ultrarapide nécessaire à la restitution de l'image est alors réalisé dans le domaine optique, en utilisant une lentille conique dont la focale varie avec la hauteur le long de l'axe et qui est balayée très rapidement le long de cet axe par le faisceau optique, au moyen d'un miroir en oscillation rapide synchrone du retour des échos.

Une deuxième méthode, utilisant trois barrettes de transducteurs, a été réalisée par la même équipe [19]. Le procédé de "retournement du temps" décrit précédemment est remplacé ici par une technique d'inversion de phase, valable pour des signaux échographiques suffisamment longs.

La phase des signaux électroniques délivrés par les transducteurs de la première barrette est inversée au moyen d'une opération de multiplication du signal $A \cos \left(\left(\omega t+\Delta \phi\left(x_{n}\right)\right)\right.$ avec un signal de référence haute fréquence qui oscille en $\cos (\Omega t)$. Un filtre passe bas permet alors de sélectionner la composante basse fréquence $(\Omega-\omega)$ dont la modulation de phase est alors inversée par rapport à celle du signal échographique et varie selon $\cos \left((\Omega-\omega) t-\Delta \phi\left(x_{\mathrm{n}}\right)\right)$. On obtient ainsi, en appliquant ces signaux à une deuxième barrette de transducteurs, une onde convergente de fréquence $(\Omega-\omega)$ pour chaque onde ditergente de fréquence $\omega$. L'effet de zoom permettant la focalisation de toutes ces ondes convergentes au niveau d'une troisième barrette fixe s'obtient en modifiant au fur et à mesure du retour des échos la fréquence $\Omega$ de l'onde de référence.

Ces techniques, jusqu'à présent, n'ont pas donné des résultats spectaculaires en imagerie médicale, du fait d'une part des difficultés technologiques rencontrées pour réaliser plusieurs barrettes de qualité, et, d'autre part, de la limitation apportée à la résolution en profondeur par l'utilisation de signaux sinusoïdaux relativement longs. C'est cependant un domaine qui reste ouvert en imagerie médicale.

A côté de ces techniques, les procédés dits de synthèse de faisceau (beam forming), utilisés en imagerie sous-marine, connaissent un développement important. Ces techniques ont été mises au point non pour reconnaître des ondes sphériques, mais pour reconnaître l'équivalent de ces ondes lorsque les cibles observées sont situées en champ lointain. On sait alors qu'on peut assimiler une onde sphérique à une onde plane au niveau de l'ouverture de réception. Le principe utilisé consiste à réaliser, à partir des signaux délivrés par une barrette de $N$ transducteurs récepteurs, $P$ signaux électriques dont le contenu respectif est celui de $P$ ondes planes d'incidences différentes arrivant sur la barrette. La réception d'une onde plane oblique d'angle d'incidence $\theta_{\mathrm{p}}$, se fait en plaçant derrière chaque transducteur une ligne à retard de valeur.

$$
\Delta \tau_{\mathrm{n}, \mathrm{p}}=\frac{\sin \theta_{\mathrm{p}}}{c} x_{\mathrm{n}} .
$$

On redresse ainsi sélectivement le front d'onde d'incidence $\theta_{p}$, et en sommant tous ces signaux remis en phase, on obtient à la sortie du sommateur le contenu échographique du faisceau réfléchi dans la direction $\theta_{p}$ (voir Fig. 13). L'observation de ce signal au cours du

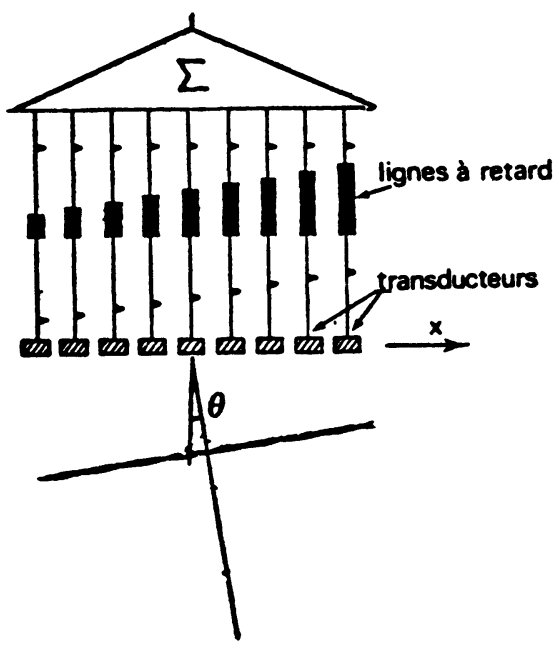

Fig. 13. - Principe de « Beam Forming ». Parmi les ondes planes arrivant sur la barrette de transducteurs, seule l'onde plane d'incidence $\theta$ donne un signal intense à la sortie du sommateur.

[Beam forming. A plane wave with an angle of incidence $\theta$ gives a strong electrical signal.]

temps donne l'image de la ligne échographique d'incidence $\theta_{\mathrm{p}}$. Pour réaliser simultanément l'image de $P$ faisceaux d'incidences différentes, il faut disposer de $P \times N$ lignes à retard, ou alternativement de $N$ lignes à retard ayant chacune $P$ sorties. De tels dispositifs 
fonctionnent couramment en imagerie sous-marine. Pour étendre ces techniques à la détection d'ondes sphériques d'incidence et de courbures variées, il faut disposer, en plus, de nombreuses lignes à retard capables de rattraper la courbure des ondes sphériques. La quantité de lignes à retard à mettre en œuvre est trop importante pour que de tels dispositifs aient été réalisés.

Une fois encore, cependant, ces procédés de décodage ont pu être simplifiés en utilisant des signaux d'illumination suffisamment longs. On peut alors remplacer les retards par des circuits déphaseurs obtenus très simplement (Fig. 14) : le signal délivré

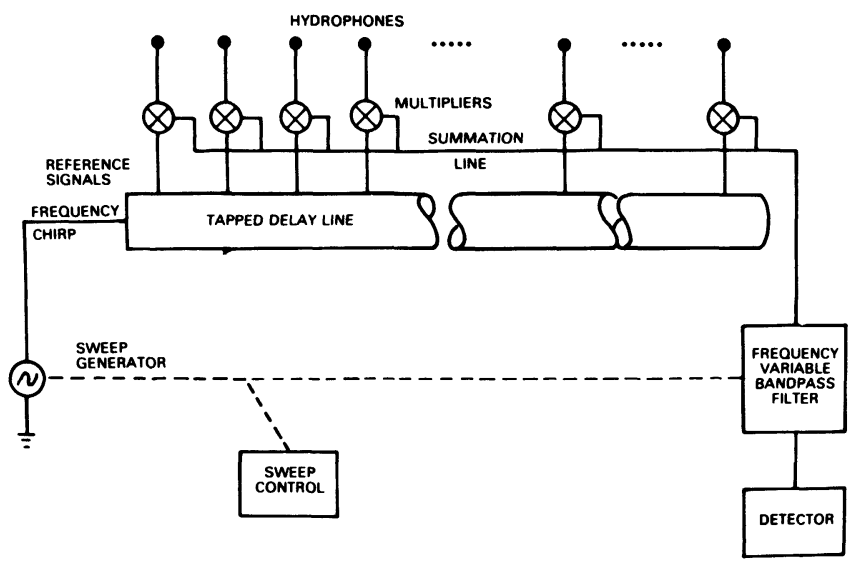

Fig. 14. - « Beam forming » obtenu par une ligne à retard à sortie multiple. La loi de retard linéaire utilisée dans la figure 13 est remplacée par une loi de déphasage linéaire. Les déphasages des signaux sortant des hydrophones sont obtenus par multiplication avec des signaux de référence qui sont pris à chacune des sorties de la ligne à retard. En injectant dans cette ligne à retard un signal de fréquence fixe, on obtient une loi de déphasage qui correspond à un angle d'incidence $\theta$ particulier. En balayant cette fréquence, on balaye les différentes incidences.

[Beam forming obtained through a multiple exit delay line.]

par chaque transducteur est multiplié par un signal de référence particulier et additionné aux autres signaux. Ce signal de référence est obtenu en injectant un signal sinusoïdal le long d'une ligne à retard à sorties multiples, produisant ainsi devant chacun des transducteurs le déphasage linéaire du signal de référence, nécessaire à la reconstruction d'une onde plane d'incidence particulière. Cette technique extrêmement élégante permet aussi de focaliser (détection d'ondes sphériques). Il suffit alors de synthétiser une loi de déphasage quadratique, en fonction de la position des transducteurs. On injecte alors non pas une sinusoïde dans la ligne à retard, mais un signal modulé linéairement en fréquence $\mathrm{e}^{i \alpha t^{2}}$ (" chirp "). En se déplaçant le long de la ligne à retard, ce signal sera perçu par chacun des transducteurs comme ayant une fréquence variant linéairement avec la position des transducteurs, et donc comme un signal sinusoïdal dont la phase instantanée varie quadratiquement avec la position. La distance focale de ce système peut être contrôlée en changeant au cours du temps le balayage en fréquence du signal de modulation. Une telle technique a été mise en œuvre à Standford par l'équipe de Kino [20]. Différentes techniques ont été utilisées pour retarder les signaux modulés en fréquence, incluant les lignes à ondes de surface, les CCD, les registres à décalages [21], les lignes à retard à sorties multiples du type cellules $\mathrm{LC}$, et enfin l'utilisation de mémoires mortes ROM. De tels dispositifs ont été étudiés en laboratoire mais n'ont pas encore débouché sur l'application industrielle.

2.4 TRAITEMENT ÉCHOGRAPHIQUE SÉQUENTIEL. Jusqu'à présent, les procédés d'exploration séquentielle des plans de coupe d'èchographie B ont donné des résultats beaucoup plus exploitables par le médecin. Dans ces systèmes où l'on choisit d'explorer séquentiellement un plan en utilisant une illumination sélective, l'emploi d'une sonde ultrasonore unique émettrice-réceptrice permet à chaque tir ultrasonore de réaliser l'image d'une ligne de l'objet, en tenant compte du temps d'arrivée des échos. Les appareils d'échographie classique (Fig. 15) font appel à une sonde plaquée directement sur la peau du patient. En asservissant le déplacement de la sonde, au moyen d'un jeu de potentiomètres, à celui du spot d'un oscilloscope à mémoire, on réalise alors l'image d'un plan de coupe en mode B. Dans les appareils actuels, ces images sont stockées dans une mémoire numérique et elles sont relues à une cadence vidéo sous forme d'une image dont les niveaux de gris sont reliées logarithmiquement à l'amplitude des échos reçus.

La résolution axiale de ces dispositifs est excellente puisqu'elle est simplement reliée à la durée de l'écho reçu $(\sim \mathrm{mm})$. Le défaut essentiel de la plupart de ces appareils est la mauvaise résolution latérale liée à la largeur du faisceau ultrasonore qui, en l'absence de focalisation, est souvent supérieure au demi-centimètre. Il faut cependant remarquer que, bien que la largeur du faisceau soit importante, la résolution latérale apparente des coupes échographiques est bien meilleure lorsque l'image est obtenue en superposant de nombreuses lignes échographiques prises sous différents angles (technique de l'échographie manuelle). Ce phénomène est lié à l'intégration spatiale des informations obtenues au cours du temps dans la formation de l'image, l'écho provenant d'une cible située à l'intersection de plusieurs faisceaux apparaissant sur l'image de façon beaucoup mieux focalisée. Cette technique est d'ailleurs reprise actuellement dans les expériences d'échotomographie digitale (voir p. 36).

2.4.1 Sondes focalisantes à symétrie circulaire. Malgré cette relative amélioration de la résolution latérale par intégration, on s'est vite intéressé à la réalisation de sondes focalisantes. Un des problèmes 

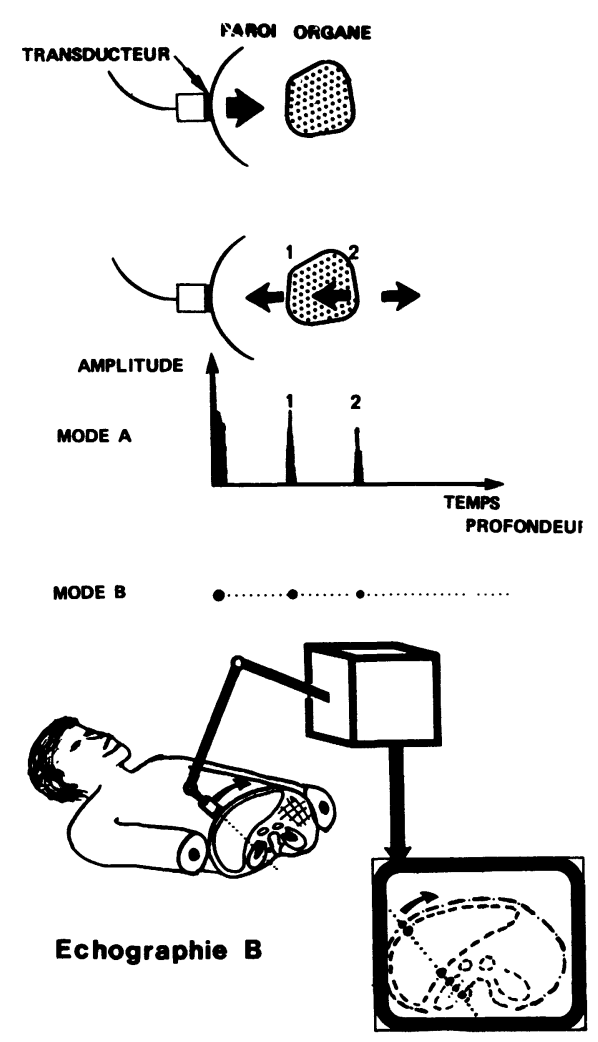

Fig. 15. - Réalisation d'une échographie. Le transducteur émet une impulsion ultrasonore qui se réfléchit partiellement sur les différentes surfaces rencontrées. Le même transducteur est utilisé comme récepteur. Les échos sunt présentés sur un oscilloscope en mode A (amplitude de l'écho en fonction du temps qui s'est écoulé après l'émission, c'est-à-dire en fonction de la profondeur), ou en mode B (échelle de temps identique mais l'amplitude de l'écho sert à moduler la brillance du spot). La ligne, où sont présentés les échos en mode $\mathrm{B}$, a la même orientation que la ligne de tir ultrasonore. Lorsque la sonde est déplacée, dans un plan fixé, les échos dessinent de proche en proche sur l'écran les contours des organes.

[Principles of A and B mode imaging.]

qui se pose alors est lié à la taille des sondes qui sont difficilement applicables par contact direct sur la peau. On s'est alors tourné vers des systèmes de sondes à rotation sectorielle mécanique, qui sont mises en mouvement dans un liquide de couplage en contact avec la peau au moyen d'une poche transparente aux ultrasons. Ces secteurs mécaniques, qui peuvent donner des cadences d'images allant jusqu'à 15 images par seconde, connaissent actuellement un grand succès. L'image sectorielle ainsi formée est stockée dans une mémoire numérique et des techniques de conversion de standard et d'interpolation permettent de la relire sous forme d'une image vidéo. La réalisation d'un pinceau ultrasonore assez fin sur une grande profondeur n'est cependant pas une opération aisée. Les transducteurs à focalisation géométrique qui sont généralement obtenus à partir de céramiques piézoélectriques moulées en forme de coquille sphérique donnent une bonne résolution latérale à la distance focale. Elle est de l'ordre de :

$$
\Delta r_{6 \mathrm{db}} \simeq \lambda N
$$

où $N$ est le nombre d'ouverture du transducteur qui mesure le rapport $(F / D)$ entre la focale et le diamètre du transducteur.

Cette résolution latérale n'est maintenue à $50 \%$ près que sur une zone dont la profondeur est égale à

$$
\Delta z \simeq 7 \lambda N^{2} .
$$

A $3 \mathrm{MHz}$ un transducteur ouvrant à $2(F / D=2$ et $\lambda=0,5 \mathrm{~mm})$ a une profondeur de focalisation correcte limitée à $20 \mathrm{~mm}$. Pour remédier à ce problème, certains constructeurs d'appareils médicaux (Kretz, Phillips) ont développé des sondes multiples rotatives formées de plusieurs transducteurs focalisant géométriquement à plusieurs distances ( 4 ou 5 transducteurs). On choisit alors la taille et la focale des transducteurs de façon à ce que les zones focales reçouvrent une grande profondeur de champ. L'ensemble des transducteurs, montés en étoile, est mis en rotation rapide au sein d'une poche liquide et l'image finale est formée par la superposition d'un nombre de sous-images égal au nombre de transducteurs. C'est l'utilisation récente de mémoires numériques d'images qui permet cette souplesse.

Une autre approche au problème de la focalisation échographique, qui tend à se développer, est celle des sondes multiples annulaires concentriques. On réalise alors, pendant la phase d'émission, un faisceau ultrasonore relativement large. Il peut être obtenu soit en excitant l'anneau central seulement, soit en excitant l'ensemble des anneaux avec un retard linéaire entre chaque anneau (lentille conique ou émission axicon) $[22,23]$. Ce n'est que pendant la phase de réception des échos qu'on cherche à réaliser une ouverture focalisante qui triera parmi tous les échos ceux qui proviennent de la ligne à afficher.

Le procédé le plus naturel pour réaliser cette ouverture focalisante consiste à utiliser derrière les transducteurs du réseau annulaire un ensemble de lignes à retard électronique dont la courbure de la loi de retard doit être adaptée à chaque instant à celle des fronts d'onde sphérique réfléchis par les cibles observées.

Pour une cible située à la distance $f$, la correction de retard apportée aux tranducteurs varie selon (voir Fig. 16)

$$
\Delta \tau_{\mathrm{f}}\left(x_{\mathrm{n}}\right)=\frac{f-\left(f^{2}+x_{\mathrm{n}}^{2}\right)^{1 / 2}}{c}=-\frac{x_{\mathrm{n}}^{2}}{2 c f} .
$$

La sommation de tous les signaux retardés donne alors un signal électrique intense uniquement pour la cible visée. Un tel effet, assimilable à une opération de zoom électronique ultrarapide, est difficile à réaliser à partir des lignes à retard variables existant actuellement. Les registres à décalage du type CCD n'ont 


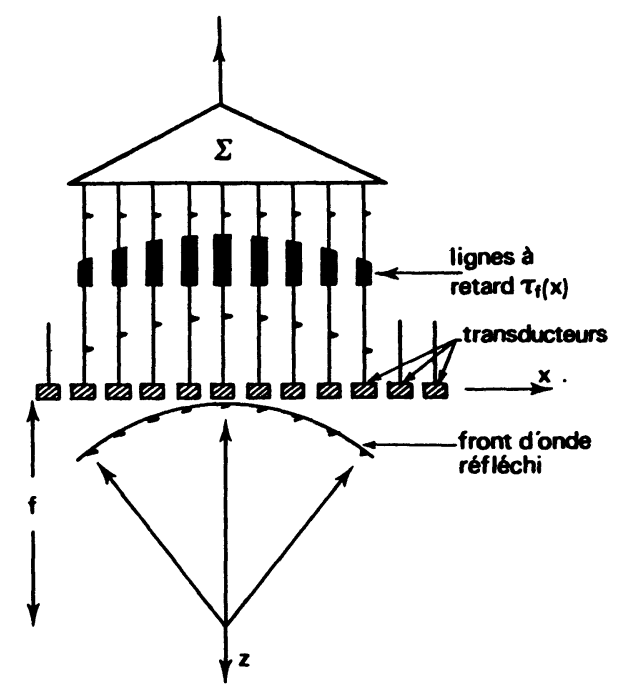

Fig. 16. - Les lignes à retard appliquent une correction de retard derrière chaque anneau transducteur (ou derrière chaque élément transducteur dans le cas des barrettes), de telle sorte que les signaux électriques délivrés par tous ces transducteurs sont remis en phase avant d'être additionnés.

[Electronic focusing in receive mode with adapted delay lines.]

pas encore atteint la dynamique requise en échographie [24]. Une solution très élégante a été proposée par les chercheurs du Standford Research Institute [25]. Les lignes à retard variables sont formées d'une série de cellules LC, dont la capacité élémentaire $c$ est une capacité variable du type Varactor. En ajustant, au moyen d'une tension de référence, la valeur de cette capacité, on peut modifier à volonté l'effet retardateur. Un dispositif remarquable a été mis au point; il fonctionne à $10 \mathrm{MHz}$, et il donne des images de la thyroïde et des petits organes d'une qualité exceptionnelle.

D'autres systèmes de focalisation ont été développés qui utilisent des lignes à retard numériques, et qui ne permettent d'obtenir qu'un nombre limité de distances focales au cours d'un tir. Un bon recouvrement des zones focales permet d'obtenir de bonnes images. Dans le système développé par Arditi [26] et qui est destiné à l'exploration du sein, on obtient à $4 \mathrm{MHz}$ une résolution à $6 \mathrm{db}$, inférieure à $1,3 \mathrm{~mm}$ sur une profondeur variant de 9 à $18 \mathrm{~cm}$ (Fig. 17). En contrôle non destructif, des dispositifs du même genre ont été aussi développés. Les laboratoires Hitachi [27] ont présenté dernièrement un réseau annulaire de transducteurs concaves, fonctionnant à 3,5 et $5 \mathrm{MHz}$ en mode de cisaillement. Ce dispositif permet d'obtenir des coupes échographiques de type B d'excellente qualité dans les aciers sur une profondeur d'exploration de $100 \mathrm{~mm}$ (Fig. 18).

2.4.2 Barrettes linéaires de transducteurs. - A côté de ces techniques utilisant des sondes à symétrie annulaire et qui donnent une résolution latérale identique dans tout le plan perpendiculaire au faisceau

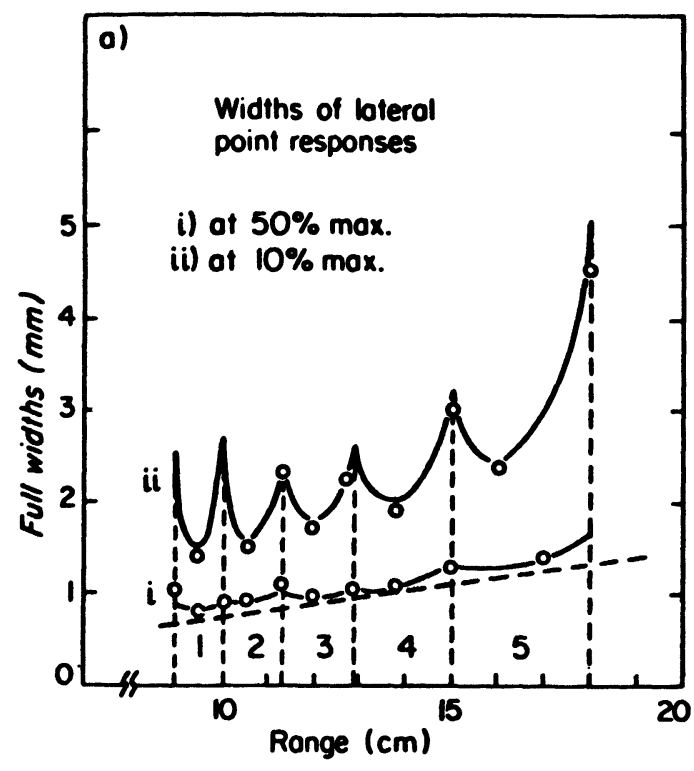

Fig. 17. - Résolutions latérales mesurées expérimentalement à partir d'un réseau annulaire de 5 anneaux fonctionnant à $4 \mathrm{MHz}$. La ligne (i) représente la résolution à $6 \mathrm{db}$, la ligne (ii) représente la résolution à $20 \mathrm{db}(26)$.

[Lateral resolution obtained by ARDITI with a 5 annular elements array working at $4 \mathrm{MHz}$.]

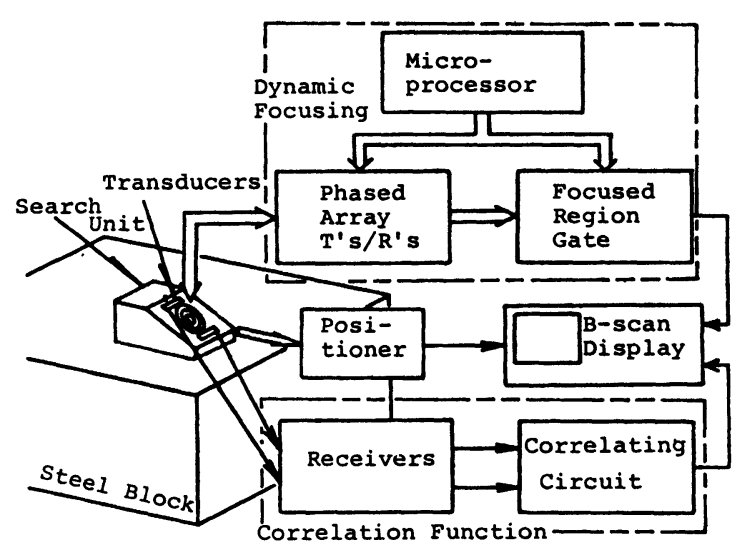

Fig. 18. - Dispositif de focalisation dynamique développé par la firme Hitachi. Il fonctionne en mode de cisaillement.

[Dynamic focusing transducer developped by Hitachi, it works in shear mode.]

ultrasonore, on a cherché à développer des sondes échographiques à partir de barrettes de transducteurs. Une commutation électronique rapide permet alors d'atteindre une cadence d'images plus élevée (50 à 100 images par seconde) qui est nécessaire dans certaines applications (cardiologie, examen des foetus, etc...). En associant à ce balayage électronique un procédé de focalisation, on peut alors bénéficier à la fois d'une imagerie à haute résolution et du temps réel. Les problèmes qui se posent sont, d'une part, la réalisation de barrettes de transducteurs de qualité (voir Annexe 3), et, d'autre part, la mise en ouvre d'une électronique de commutation et de focalisation 
par lignes à retard identiqueś à celles décrites pour les sondes annulaires. On a réalisé ainsi d'excellentes sondes à balayage électronique linéaire, qui, du fait de l'absence de partie mécanique fragile (moteurs) sont relativement bon marché tout en donnant d'excellentes performances. Le marché est dominé par l'industrie japonaise où les marques Toschiba, Aloka et Hitachi sont les mieux placées. Les barrettes fonctionnent à des fréquences comprises entre 3 et $5 \mathrm{MHz}$, et les meilleures résolutions latérales obtenues dans l'eau à $3 \mathrm{MHz}$ sont de l'ordre de $2 \mathrm{~mm}$ (Fig. 19).

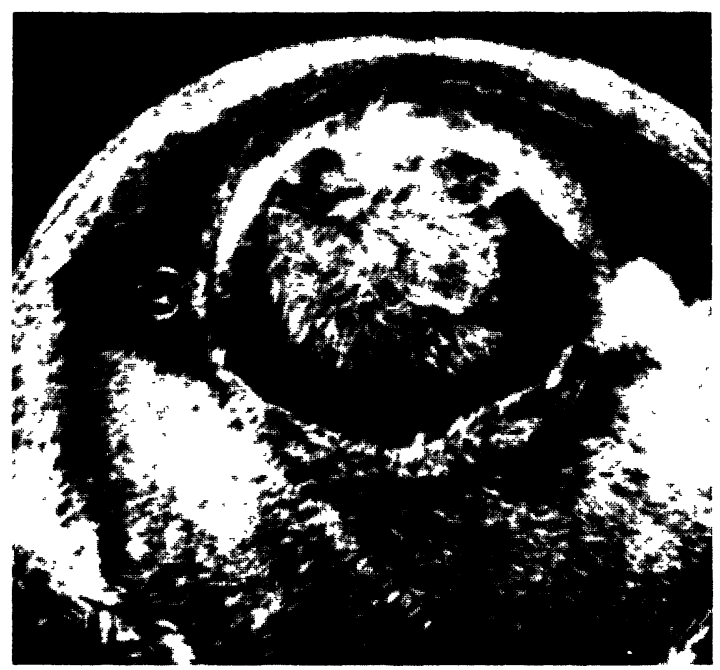

a)

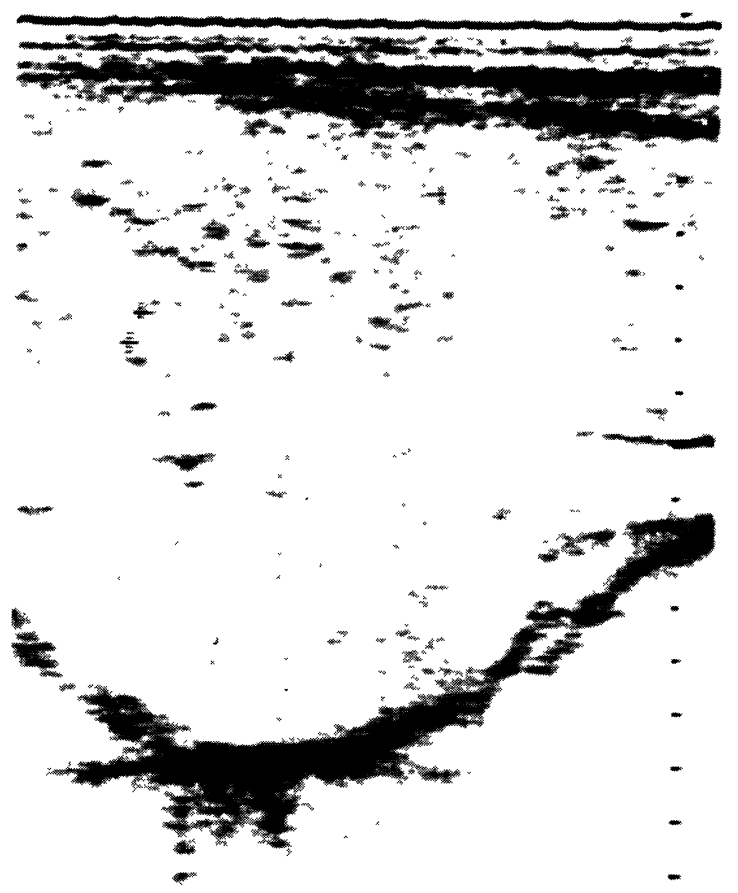

b)

Fig. 19. - a) Coupe échographique d'une tête de foetus obtenue en mode B. $b$ ) Coupe d'un foie obtenue au moyen d'une barrette linéaire de transducteurs. On observe au sein $\mathrm{du}$ foie des coupes longitudinales et transverses de veines. Le rebord inférieur du foie est bien visible.

[a) B mode image of a foetal head. b) B mode image of a liver obtained with a linear array.]
Ces barrettes présentent cependant un inconvénient important comparé aux sondes annulaires. La focalisation d'origine électronique n'a lieu que dans la direction de la barrette (résolution azimutale). Dans la direction perpendiculaire, à la fois à la barrette et au faisceau ultrasonore (résolution en élévation), la résolution est généralement beaucoup moins bonne. On a cherché à remédier à ce défaut en accolant des lentilles cylindriques de caoutchouc ou d'araldite à ces barrettes. On obtient alors une bonne résolution latérale en élévation, mais uniquement autour d'une distance focale privilégiée. La tranche explorée par cette barrette n'a pas une épaisseur uniforme et, dans certains cas, les images médicales sont fortement dégradées. Une solution a été proposée par le groupe Siemens [28] pour remédier à cet inconvénient. Elle consiste à regrouper systématiquement autour d'une barrette deux autres barrettes identiques faisant un angle de quelques degrés avec la première barrette. Ce dispositif est utilisé à l'émission et permet d'obtenir une tranche d'épaisseur relativement uniforme (résolution en élévation à $6 \mathrm{db}$ de $4 \mathrm{~mm}$ sur $18 \mathrm{~cm}$ ) (voir Fig. 20).

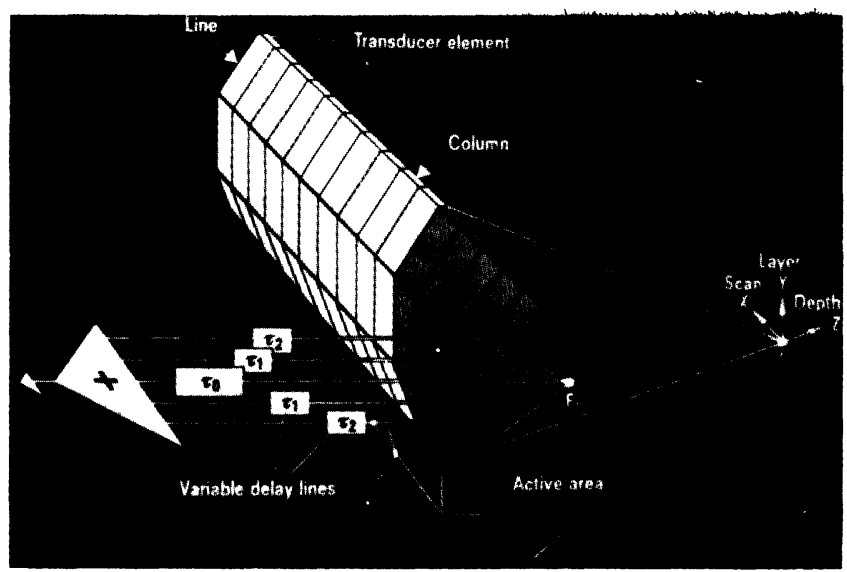

Fig. 20. - Dispositif à 3 barrettes développé par Siemens.

[Combined linear arrays (Siemens).]

Parallèlement au développement des barrettes à balayage linéaire qui sont nécessairement de grande taille pour donner l'image d'un champ de dimension raisonnable $(12$ à $20 \mathrm{~cm})$, des barrettes de dimension beaucoup plus petite ont été réalisées pour les applications cardiaques. On utilise alors un balayage sectoriel du plan étudié, plutôt qu'un balayage linéaire. L'ouverture maximale de ces barrettes pour l'application en cardiologie est limitée par la distance intercostale à $25 \mathrm{~mm}$. Les fréquences maximales d'utilisation sont limitées par l'atténuation ultrasonore dans le cour à $5 \mathrm{MHz}$ [29-31].

Le principe du fonctionnement de ces barrettes est similaire à celui du "Beam forming " en imagerie sous-marine, mais il est utilisé ici dès l'émission. En appliquant une impulsion d'émission décalée linéairement dans le temps d'un transducteur à l'autre, 

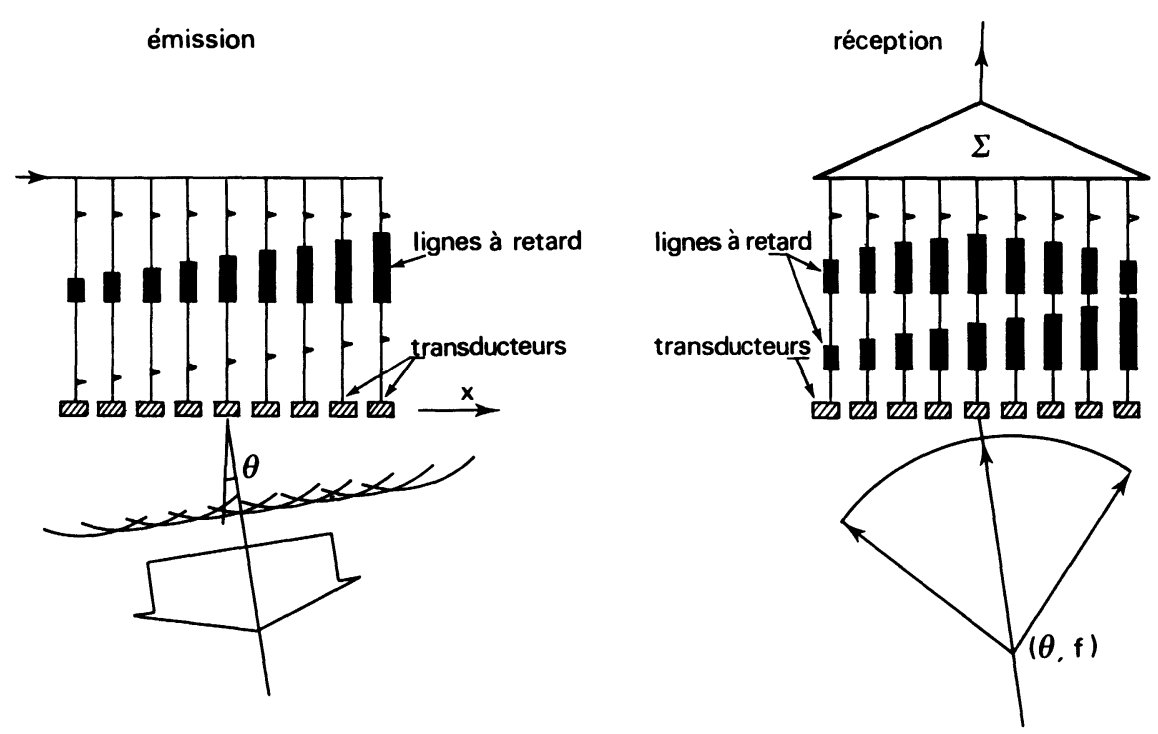

Fig. 21. - Balayage sectoriel et focalisation électronique à partir d'une barrette de transducteurs. L'impulsion d'émission est décalée linéairement dans le temps, d'un transducteur à l'autre; l'angle $\theta$ du faisceau avec la normale à la barrette dépend de la loi de retard. A gauche : émission. A droite : réception.

[Electronic beam steering in transmit mode and oblique focusing in receive mode.]

on oriente le faisceau d'émission d'un angle $\theta$ avec la normale à la barrette (Fig. 21) selon une loi en

$$
\Delta \tau_{\theta}\left(x_{n}\right)=\frac{\sin \theta}{c} x_{n},
$$

qu'on peut modifier d'un tir à l'autre. On utilise alors à la réception un procédé de focalisation oblique qui combine à la fois les corrections de retard linéaire et quadratique nécessaires pour reconnaître les cibles orientées suivant l'angle $\theta$

$$
\Delta \tau_{\theta, \mathrm{f}}\left(x_{\mathrm{n}}\right)=\frac{\sin \theta}{c} x_{\mathrm{n}}-\frac{x_{\mathrm{n}}^{2}}{2 c f} .
$$

Il est important de préciser que ce type de barrette est décrit outre Atlantique sous le nom de «Phased Array ». Cette terminologie vient du fait que très souvent les lignes à retard peuvent être remplacées par des circuits déphaseurs. Il faut noter ici que la technologie de ces barrettes est plus délicate que celle des barrettes à balayage linéaire. Si l'on veut pouvoir obtenir des ouvertures angulaires importantes (un champ de $90^{\circ}$ est une ouverture type), il est en effet nécessaire d'avoir un réseau de transducteurs ayant un pas inférieur ou égal à la demi-longueur d'onde, afin d'éviter l'apparition de lobes de réseau dans l'ouverture angulaire. Ces lobes de réseau limitent très fortement la dynamique du système. On doit obtenir des densités d'éléments transducteurs d'au moins 32 éléments par centimètre à $2,5 \mathrm{MHz}$.

La première barrette sectorielle réalisée par Somer [30] avait 21 éléments au pas de $\lambda / 2$, mais elle fonctionnait seulement à $1,3 \mathrm{MHz}$ et elle ne focalisait pas. Le système développé par Thurstone [31] utilisait une ouverture de $25 \mathrm{~mm}$ à $2,25 \mathrm{MHz}$. Cinq distances focales étaient synthétisées à la réception. Le nombre des éléments était cependant limité à 16 , ce qui entraînait un très fort niveau de lobes secondaires.

Le système développé par Larson et son équipe [32] chez Hewlett-Packard est réalisé à partir d'une barrette de $20 \mathrm{~mm}$ d'ouverture formée de 64 éléments fonctionnant à 2,5 $\mathrm{MHz}$. La résolution azimutale à $6 \mathrm{db}$ à la profondeur de $7 \mathrm{~cm}$ est de $2,5 \mathrm{~mm}$, la résolution axiale est de $1 \mathrm{~mm}$ et la résolution en élévation de 3,5 mm (Fig. 22). La technologie très soignée de cette barrette de transducteurs permet d'obtenir un niveau de lobes secondaires inférieur à $65 \mathrm{db}$, et des échos très propres sans traînée. D'excellentes images sont obtenues à partir de ce dispositif qui couvre un champ de $90^{\circ}$ sur une profondeur de $24 \mathrm{~cm}$. L'orientation et la focalisation du faisceau sont obtenues au moyen d'une technique mixte faisant appel d'une part à un nombre réduit de lignes à retard de faible bande passante et d'autre part à une technique de déphasage par hétérodynage. Un tel procédé proposé par différents auteurs [33, 34] repose sur la possibilité de traiter l'enveloppe du signal échographique indépendamment de sa porteuse. On peut en effet, pour un signal échographique de bande passante $B$ limitée $\left(B<F_{0}\right)$, écrire ce signal sous la forme

$$
e(t)=a(t) \cos \left(2 \pi F_{0} t+\theta\right) .
$$

Pour retarder ce signal d'un temps $\tau$, il suffit d'une part de retarder l'enveloppe $a(t)$ qui est un signal basse fréquence $(F<B / 2)$ et d'autre part de déphaser la porteuse d'une quantité $\phi_{0}(\tau)=2 \pi F_{0} \tau$ (module $2 \pi$ ). On obtient ainsi le signal translaté

$$
s(t-\tau)=a(t-\tau) \cos \left(2 \pi F_{0}(t-\tau)+\theta\right) .
$$



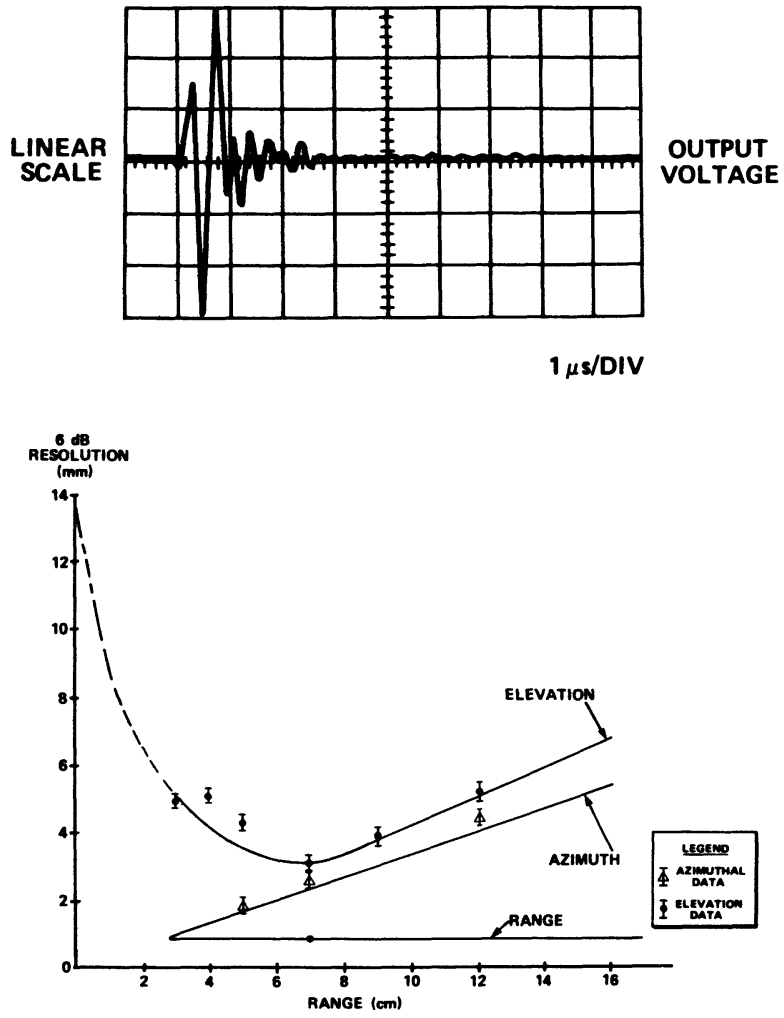

Fig. 22. - Résultats expérimentaux du dispositif développé par Hewlett Packard. En haut : réponse échographique. En bas : résolutions azimuthales, en élévation et axiale à $6 \mathrm{db}$.

[Experimental results obtained from the phased array developped by Hewlett Packard.]

On peut aussi effectuer l'opération de déphasage en modifiant la fréquence de la porteuse. Un hétérodynage du signal par un oscillateur de fréquence $F_{1}$ et de phase ajustable $\phi_{1}(\tau)=2 \pi\left(F_{1}-F_{0}\right) \tau$ donne alors un signal de fréquence intermédiaire $F_{0}-F_{1}$ correctement retardé. L'architecture d'un tel dispositif est présentée sur la figure 23 , elle nécessite de traiter séparément les deux composantes en quadrature du signal $e(t)$.

Parallèlement à ces techniques purement analogiques, la possibilité de translater la fréquence centrale échographique vers des fréquences intermédiaires plus basses, sans perdre aucune information sur le signal échographique, permet d'envisager la conversion analogie digitale de ces signaux hétérodynés à des fréquences d'échantillonnage relativement faibles (1 à $2 \mathrm{MHz}$ ). C'est dans cette voie que s'orientent actuellement plusieurs constructeurs [34]. Le traitement de focalisation est alors réalisé de façon complètement digitale.

2.4.3 Traitement du signal en échographie Doppler et en caractérisation tissulaire. - Dans toutes les techniques d'échographie séquentielle décrites précédemment, des traitements du signal de plus en plus sophistiqués sont utilisés pour focaliser et pour orienter le faisceau d'exploration. Il reste cependant
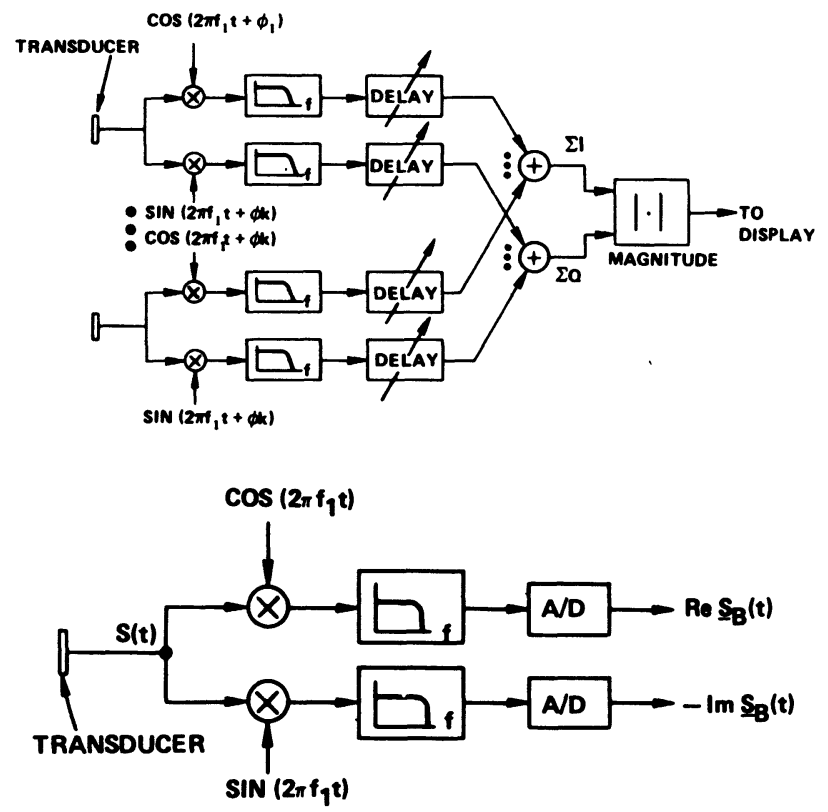

$S(t)=S_{B}(t) \exp \left[j 2 \pi f_{0} t\right]$
$S_{B}(t)=A(t) \exp [-j \phi(t)]=I(t)-j Q(t)$

Fig. 23. - Phased-Array. - En haut : focalisation par déphasage de la porteuse et décalage de l'enveloppe. En bas : technique de digitalisation basse fréquence utilisant un héterodynage préalable. On obtient les deux composantes en quadrature du signal analytique.

[Phased array. - Focusing principles (above). - Low frequency $A / D$ conversion using a heterodyning circuit (below).]

que l'information échographique, une fois refocalisee, est traitée de façon relativement simple avant sa présentation sous forme d'image vidéo. Une démodulation d'amplitude (détection d'enveloppe) du signal HF est systématiquement effectuée. L'information de fréquence instantanée est actuellement négligée, sauf dans le cas bien précis de l'échographie Doppler.

- On sait qu'un faisceau ultrasonore dirigé à travers la peau sur un vaisseau sanguin est diffusé par les globules rouges. Du fait du mouvement de ces globules on observe une différence de fréquence entre le faisceau d'exploration et le faisceau rétrodiffusé par le sang. Pour une cible mobile unique, ce décalage fréquentiel $\Delta F$ est relié à la vitesse $v$ de la cible à travers la formule

$$
\Delta F=\frac{2 F v \cos \theta}{c} .
$$

$\theta$ est l'angle que fait le faisceau d'exploitation avec la direction de l'écoulement. Le facteur 2 provient du fait qu'en échographie la cible joue tour à tour le rôle de récepteur et d'émetteur ultrasonore.

- Différents problèmes se posent en échographie Doppler pour mesurer localement la vitesse d'écoulement et pour en déduire, par une intégration sur la section du vaisseau, le débit sanguin. 
- En raison de la faible dimension des cibles diffusantes les échos du sang sont très peu intenses. Leur amplitude est pratiquement inférieure de 50 à $60 \mathrm{db}$ à celles des échos des parois artérielles. En échographie B classique ils n'apparaissent pas sur les images d'artères. Des circuits d'élimination des échos fixes sont donc souvent nécessaires pour recueillir le signal des diffuseurs mobiles.

- La réflexion du signal ultrasonore sur ces diffuseurs est du type "diffusion de Rayleigh ", qui prévoit une énergie rétrodiffusée en $F^{4}$. Le choix de la fréquence optimale résulte cependant d'un compromis avec les effets de l'atténuation des tissus (qui croît avec la fréquence). Les valeurs couramment utilisées vont de 5 à $15 \mathrm{MHz}$ pour les vaisseaux superficiels et de 2 à $5 \mathrm{MHz}$ pour les vaisseaux profonds.

- Les mesures sont perturbées par le grand nombre de globules rouges, de vitesses différentes, observés simultanément. Le signal Doppler s'étend habituellement sur toute une gamme spectrale. Une analyse spectrale détaillée de ce signal serait nécessaire, mais on lui préfère généralement une estimation de la fréquence centrale Doppler définie par

$$
\bar{F}=\frac{\int_{0}^{\infty} S(F) F \mathrm{~d} F}{\int_{0}^{\infty} S(F) \mathrm{d} F},
$$

où $S(F)$ est le spectre Doppler.

- Un autre problème est lié à la difficulté de mesurer simultanément avec précision la position et la vitesse d'une cible (relation d'incertitude). Enfin pour obtenir une information quantitative sur la vitesse du sang, il faut pouvoir déterminer avec précision l'angle $\theta$, ce qui nécessite parallèlement au traitement Doppler, une visualisation de l'orientation des vaisseaux.

- 2 types de dispositifs ont été développés jusqu'à présent.

- Les appareils à émission continue. Ce sont les plus répandus. Ils n'ont pas de résolution en profondeur et on les utilise surtout pour les vaisseaux peu profonds : carotides, artères fémorales. Les fréquences sont de l'ordre de $10 \mathrm{MHz}$. Du fait de l'émission continue, on est obligé d'utiliser 2 céramiques distinctes à l'émission et à la réception. La comparaison des deux fréquences d'émission et de réception donne un signal BF qui peut être écouté par le médecin $(\Delta F<5 \mathrm{kHz})$, ou enregistré graphiquement.

- Les dispositifs de Doppler pulsé. L'utilisation d'une émission pulsée peut donner une information de vitesse dans des zones de faible dimension situées à des profondeurs réglables. En utilisant le même transducteur en émission-réception, on isole les échos d'une certaine profondeur au moyen d'une fenêtre d'observation de dimension et d'origine réglable [35].
La mesure de la fréquence moyenne du signal Doppler peut être réalisée de plusieurs façons. Un des procédés les plus employés utilise un comptage des passages à zéro au sein de la fenêtre. (Le nombre de passages à zéro est relié à la fréquence quadratique moyenne.) C'est une technique très simple, qui est cependant bruiteuse et peu précise. D'autres procédés utilisent des banques de filtres ou des analyseurs du type FFT. On peut aussi évaluer, au moyen de circuits analogiques, le numérateur et le dénominateur de la formule 14 . Un circuit diviseur délivre alors la fréquence moyenne [36].

- L'avantage indéniable des techniques de Doppler pulsé a cependant été longtemps mal exploité dans l'exploration des vaisseaux profonds, du fait de la difficulté à visualiser la direçtion du faisceau Doppler. La nécessité de coupler des dispositifs Doppler pulsés à fenêtres multiples à des échographes de type $B$ est apparue évidente.

- A l'heure actuelle, plusieurs firmes développent des systèmes «Duplex » qui associent à l'image échographique donnée par une sonde sectorielle mécanique un transducteur auxiliaire fonctionnant en mode Doppler pulsé. Ce transducteur peut être déplacé manuellement dans différentes régions repérées sur l'image sectorielle. Des techniques plus récentes s'orientent vers l'utilisation des transducteurs d'une même barrette pour produire à la fois l'image échographique et une ligne d'exploration Doppler pouvant être déplacée électroniquement au sein de l'image. L'exploration des vaisseaux abdominaux de l'adulte, ainsi que ceux du placenta et du foetus est ainsi rendue possible par ces techniques qui peuvent bien entendu être aussi utilisées en exploration cardiaque.

- Pour conclure ce paragraphe sur le traitement de signal en échographie Doppler, il faut noter que les recherches effectuées dernièrement en caractérisation des tissus ont montré que l'information de fréquence instantanée pouvait aussi être extrêmement utile pour développer une imagerie quantitative. On peut montrer, en effet, que l'évolution de la fréquence instantanée des échos le long d'une ligne échographique est directement reliée à la valeur de l'atténuation ultrasonore des tissus [37]. Cette atténuation est une fonction croissante de la fréquence, et le contenu spectral des échos se modifie avec la profondeur. On observe une chute importante de la fréquence centräle avec la profondeur qui, pour un transducteur standard de $3 \mathrm{MHz}$ de fréquence fondamentale, est de l'ordre de $500 \mathrm{kHz}$ sur l'épaisseur d'un foie sain. On peut relier ainsi l'évolution locale de cette fréquence moyenne au profil d'atténuation. Une imagerie de cette atténuation ultrasonore peut ainsi être envisagée en échographie, dans un avenir relativement proche.

2.5 RECONSTRUCTION DIGITALE TOMOGRAPHIQUE EN MODE ECHOGRAPHIQUE. - Dans ces techniques de reconstruction tomographique, on utilise une pro- 
cédure qui s'apparentè en partie à celle décrite précédemment dans le cas des barrettes de transducteurs. On réalise, à partir d'un ensemble de transducteurs, une série de tirs ultrasonores illuminant séquentiellement le plan de coupe objet sous différentes incidences. Les transcuucteurs utilisés sont généralement des transducteurs de petite dimension, et le champ rayonné peut être assimilé à un front d'onde sphérique divergent. Ces transducteurs sont utilisés en mode échographique et ils délivrent chacun à la réception un signal échographique qui intègre, à chaque instant, les échos en provenance de tous les diffuseurs rencontrés par l'onde sphérique. On associe à chaque transducteur une ligne d'échographie $\mathrm{A}$ qui provient d'un secteur angulaire très large du plan objet. Le principe de la reconstruction tomographique (qualifiée aussi souvent de focalisation synthétique) consiste, une fois stockées toutes ces lignes échographiques obtenues chacune avec une très mauvaise résolution latérale, à développer un procédé de « refocalisation » de toutes ces données.

L'opération la plus couramment utilisée pour "refocaliser " ces données est celle de la projection inverse (ou backprojection). C'est une opération beaucoup plus simple à mettre en ouvre que les techniques de focalisation décrites à partir d'une illumination par onde plane. La résolution latérale qu'on peut atteindre avec ces techniques de backprojection peut être nettement inférieure à la longueur d'onde, du fait des très grandes ouvertures angulaires sous lesquelles on observe l'objet au cours du temps. L'image d'un point source a cependant un aspect différent de celle qu'on obtient par les techniques de focalisation (voir Fig. 24).

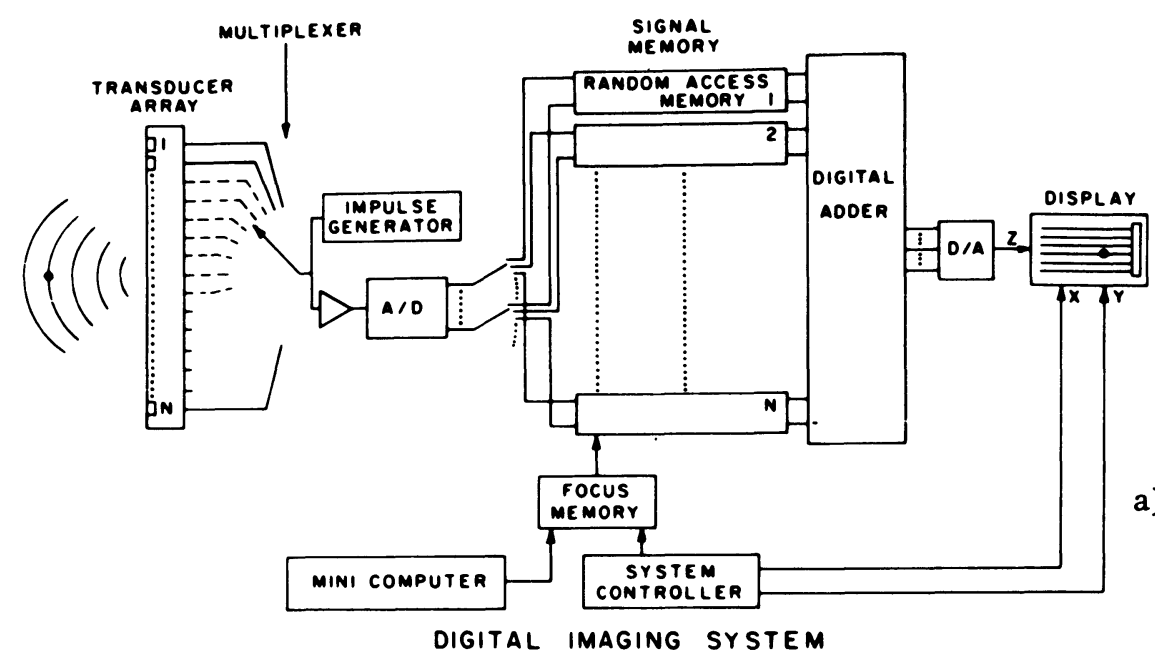

a)

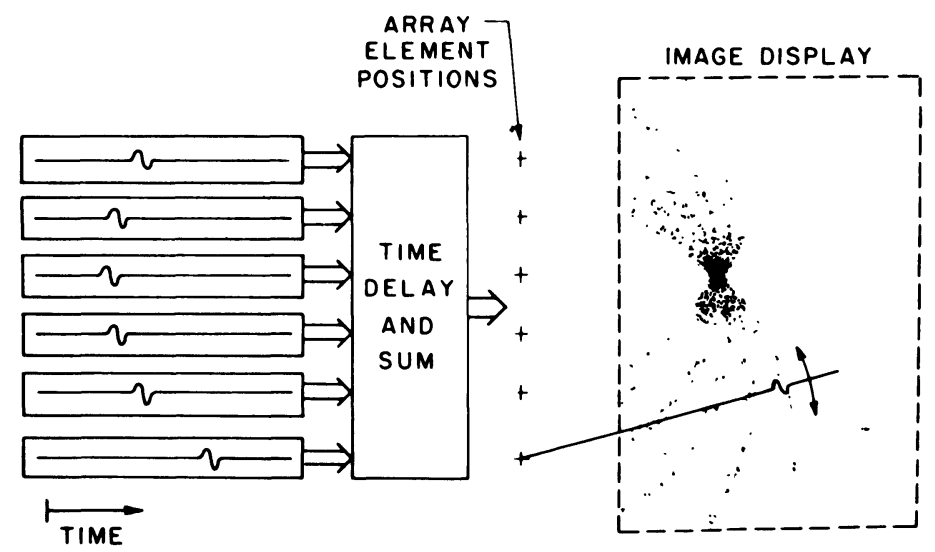

Fig. 24. - Principe du dispositif développé par Corl et Kino. La technique de projection inverse donne pour l'image d'un point une structure en étoile [35]. C'est l'intersection de la famille des arcs de cercles centrés sur les transducteurs de la barrette.

[Principles of the displaý developped by Corl and Kino [35]. The image reconstruction is obtained by back-projection.]

Le premier dispositif de ce type a été développé à Standford par l'équipe de Kino [38, 39]. Il repose sur l'utilisation d'une barrette de 32 transducteurs fonctionnant autour d'une fréquence de 3,3 $\mathrm{MHz}$. Chaque élément transducteur est utilisé en mode échogra- phique et délivre une ligne d'échographie $\mathrm{A}$, qui est amplifiée et convertie à une cadence de $16 \mathrm{MHz}$ sous forme de mots de 8 bits. Ces données sont stockées dans une mémoire vive de $1024 \times 8$ bits. Ce processus est répété tour à tour pour les 32 transducteurs 
et une mémoire RAM de 32 kilobytes est ainsi remplie. Ces données sont alors traitées par projection inverse et le résultat de cette opération est injecté dans une deuxième mémoire vive appelée mémoire focalisante. Le processus de projection inverse est effectué très rapidement et la cadence d'image qui peut être obtenue est de 30 images par seconde.

Pour illustrer la technique de projection inverse utilisée pour reconstituer la carte des sources de réflectivité $S(\mathbf{r})$ du milieu nous allons, dans un premier temps, nous intéresser à la détermination de la réflectivité en un point isolé $\mathbf{r}_{0}$ du milieu placée devant la barrette à la distance $\rho$ du premier transducteur. Pour obtenir la valeur de la réflectivité en ce point, nous observerons la ligne échographique A enregistrée par ce transducteur au temps $2 \rho / c$. Cette valeur représente, du fait de la forme d'onde sphérique émise par le transducteur, la somme des échos en provenance de tous les points sources situés sur l'arc de cercle de rayon $\rho$ contré sur l'élément en question. Ce processus est répété pour chaque point des différentes lignes échographiques enregistrées à partir des $N$ éléments de la barrette ( $\rho$ est toujours la distance entre le point isolé $\mathbf{r}_{0}$ dont on cherche la réflectivité et un élément transducteur). On additionne alors de façon cohérente ces $N$ valeurs, pour obtenir la réflectivité au point image. Chaque échographie $A$ contribue ainsi par une seule de ces valeurs à chaque point image.

Pour reconstruire tous les points de l'image de cette façon, l'amplitude de la ligne échographique évaluée au temps $2 \rho / c$ est uniformément distribuée (ou étalée) sur un arc de cercle de rayon $\rho$ dans le plan image. Cette procédure est répétée pour tous les points de chacune des $N$ lignes échographiques enregistrées, et en chacun des points du plan image les résultats sont additionnés de façon cohérente. C'est le processus qui est qualifié de projection inverse. Il ressemble par beaucoup d'égards au processus développé dans les scanners à rayon $\mathbf{X}$ fonctionnant en transmission, la différence fondamentale étant que la projection des données se fait en échographie sur des arcs de cercle, alors que dans les scanners $X$ la projection se fait le long de lignes droites [40].

Cette technique qui a été mise en œuvre en contrôle non destructif permet d'obtenir des images de défauts avec une résolution latérale de l'ordre de 2 longueurs d'onde. Des techniques de traitement non linéaire [39] permettent d'améliorer cette résolution d'un facteur 2 . Elles ne sont utilisables cependant que pour des objets contenant peu de défauts. Différentes équipes s'intéressent à des dispositifs de ce genre et particulièrement la firme Rockwell qui a mis au point derrière une barrette de 32 transducteurs entièrement digitalisée des programmes de projection inverse très rapide utilisant un "array processor ».

L'extension de ces techniques à l'imagerie à trois dimensions a été effectuée par l'équipe de Standford [41]. Il s'agit d'étudier l'image de pièces de céramique en forme de disques ou de barres cylindriques. L'onde sphérique d'illumination est réalisée au moyen d'un transducteur focalisant qui donne, au-delà de son point focal, un faisceau divergent assimilable à une onde sphérique. On obtient ainsi une intensité acoustique beaucoup plus importante qu'avec la technique utilisant des éléments transducteurs de petite dimension. A la réception, on dispose pour étudier les disques de céramiques, d'une matrice de $8 \times 8$ transducteurs, et pour les barres de céramiques de 16 anneaux formés chacun de 32 transducteurs ceinturant la région de la barre à explorer (Fig. 25). Dans les

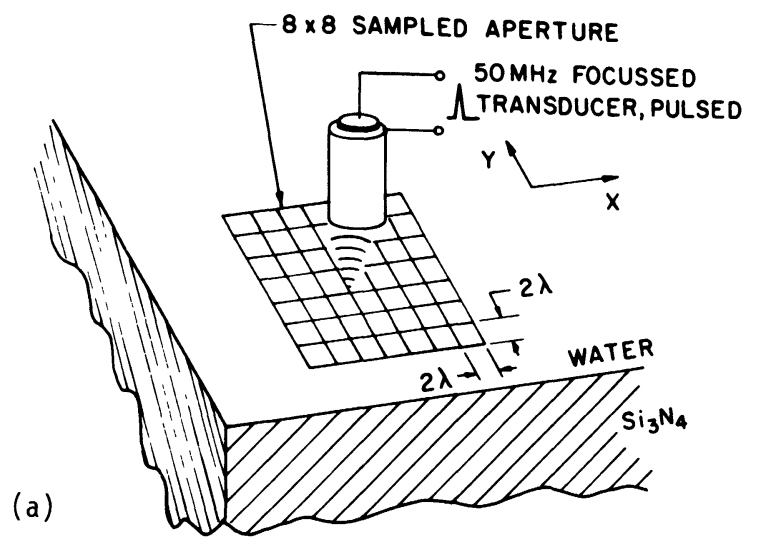

(b)

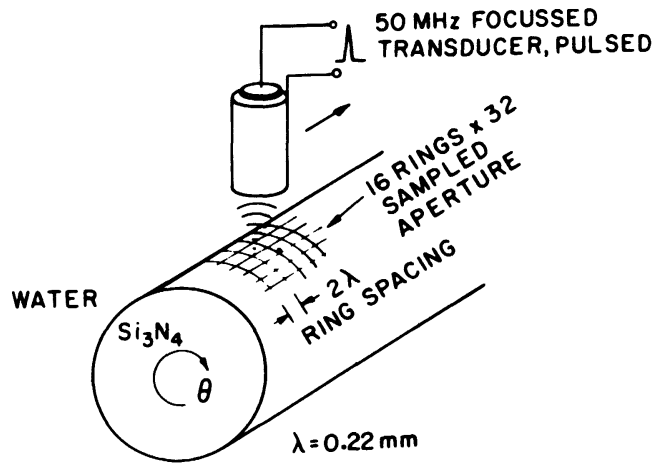

Fig. 25. - Dispositifs d'échotomographie tridimensionnelle [41] développés pour l'imagerie de blocs de céramiques. En haut : cas d'un disque de céramique. En bas : cas d'une tige cylindrique de céramique.

[3D synthetic focus system developped for ceramic imaging. - Flat disc samples (above). - Cylindrical rod samples (below).]

deux cas, la fréquence d'illumination est de $50 \mathrm{MHz}$ et la reconstruction de l'image se fait par projection inverse (Fig. 26). Des images de très bonne qualité des défauts ont été ainsi obtenues. Différentes améliorations de la technique de projection inverse peuvent être apportées, en jouant pour les unes sur l'élimination d'échos parasites, et pour les autres sur des traitements non linéaires (Fig. 27).

En contrôle non destructif, on peut rattacher à ces techniques de reconstruction des défauts, les techniques d'inversion plus rigoureuses qui tiennent 


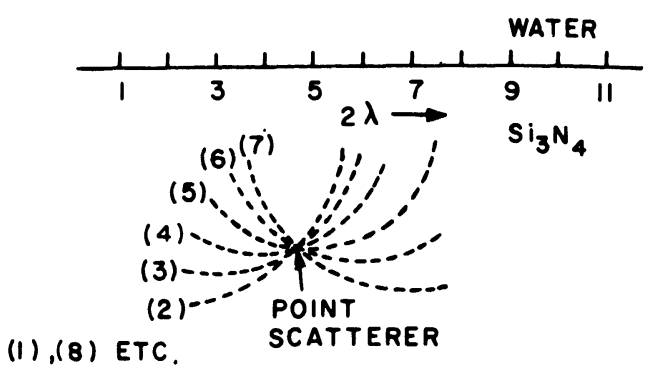

(a) SQUARE APERTURE

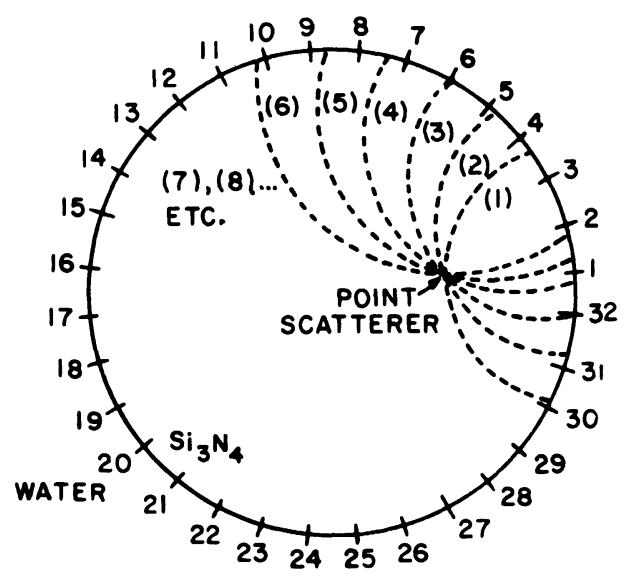

(b) CYLINDRICAL APERTURE

Fig. 26. - Principes de la projection inverse dans les deux cas de la figure 25 [41]. Dans les deux cas, la projection se fait sur des arcs de cercle autour de chacun des transducteurs récepteurs.

[Back projection principles used with figure 25 [41]. Circular back-projection is used in the two cases.]

compte des différents modes de propagation existant dans les matériaux solides. En examinant le champ diffracté par un défaut sous de nombreuses incidences, des programmes d'inversion très sophistiqués $[42,43]$ (Poffis Algorithm) permettent de reconstruire la géométrie bidimensionnelle ou tridimensionnelle de ces défauts (Fig. 28).

Dans le domaine médical, la technique proposée par Norton et Linzer [40] s'apparente à la structure annulaire retenue pour étudier les barres cylindriques de céramique. L'utilisation d'un réseau de transducteurs émetteurs-récepteurs répartis sur un anneau entourant la structure à visualiser permet, par projection inverse, d'obtenir des résolutions théoriques de l'ordre de 0,3 longueur d'onde. Jusqu'à présent cependant, la réalisation expérimentale de ces dispositifs n'a pas encore été poussée très loin.

Des versions plus simples, faisant appel à une illumination d'un plan de l'objet, par des ondes planes de grande ouverture et d'incidences variées, permettent d'obtenir en mode échographique des projections de la carte de réflectivité sous différents angles. Ces projections sont alors du type linéaire et l'opération de projection inverse est exactement celle développée pour

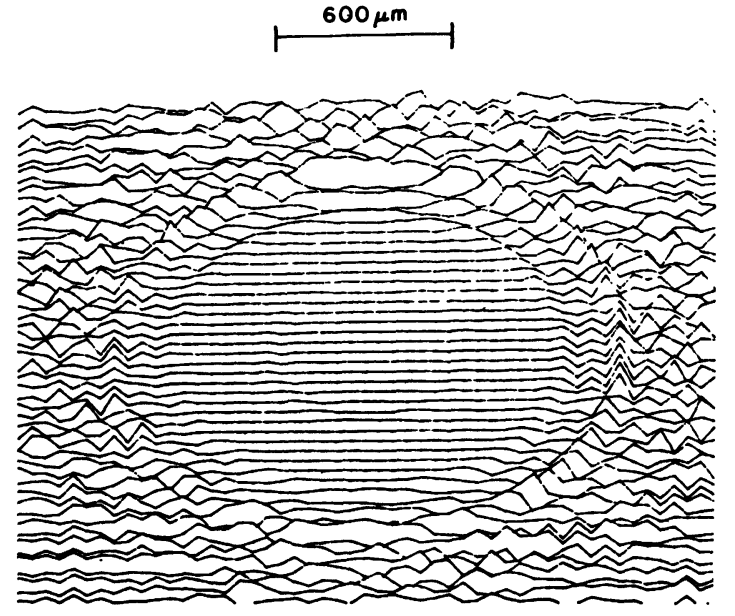

(0) CONVENTIONAL BACKPROJECTION

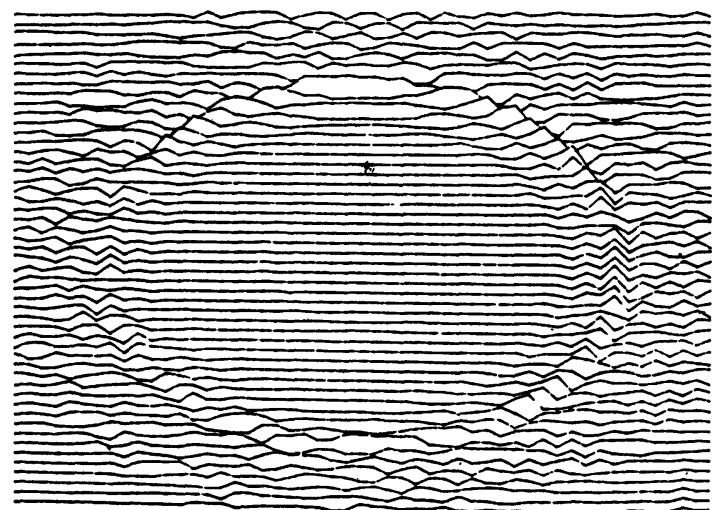

(b) SELECTIVE BACKPROJECTION

Fig. 27. - Images d'un défaut circulaire obtenues en haut par projection inverse filtrée, et en bas en utilisant des techniques de traitement non linéaire [41].

[Images of a circular void defect processed by different schemes : selective back projection (above); non linear processing (below) [41].]

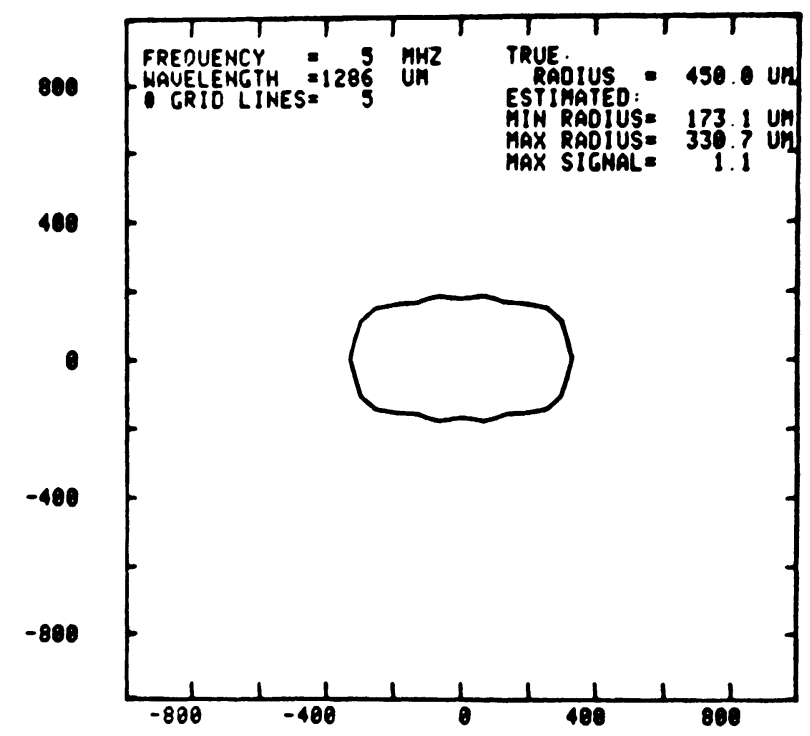

Fig. 28. - Reconstruction de la cartographie d'un défaut par la méthode Poffis [42].

[Defect reconstruction with Poffis method [42].] 
les scanners $X$; la technique de projection circulaire plus délicate à mettre en œuvre est alors évitée.

Le problème posé par la génération d'une onde plane de grande dimension n'est pas trivial. Si l'on utilise un transducteur de grande taille, on travaillera alors dans le champ proche du transducteur où la répartition de pression est très inhomogène. La méthode proposée par Hiller d'une part et par le groupe Siemens [44, 45] d'autre part, consiste à synthétiser l'écho provenant d'une onde plane de grande ouverture à partir de plusieurs tirs échographiques réalisés séquentiellement à partir d'une petite sonde collimatée qui se déplace le long d'une ouverture importante. On peut alors, en superposant les signaux échographiques préalablement détectés et filtrés provenant de $N$ tirs, obtenir par une opération de moyenne l'équivalent de l'écho redressé en provenance d'une fenêtre de grande ouverture (Fig. 29). On obtient ainsi une projection linéaire de l'objet sous un certain angle d'incidence $\theta$. On peut, en répétant cette opération de balayage sous d'autres incidences, réaliser une série de projections linéaires. Un algorithme de projection linéaire inverse donne alors la carte de réflectivité. Ces techniques permettent une résolution latérale à $6 \mathrm{db}$ de l'ordre de la demi-longueur d'onde. Les premières images obtenues in vitro semblent particulièrement bien contrastées et prometteuses.

Dans le même ordre d'idées, les deux équipes allemandes travaillant sur ce projet ont proposé une méthode d'amélioration de la résolution, qui consiste à réaliser une série de coupes échographiques en mode B d'un plan objet observées sous différentes incidences. On utilise alors une grande capacité mémoire et, en superposant correctement ces différentes images, on obtient une image finale de bien meilleure résolution. La difficulté couramment rencontrée lorsqu'on visualise des réflecteurs spéculaires mal orientés, disparaît dans ce cas et une image très précise des contours peut ainsi être obtenue.

2.6 RECONSTRUCTION DIGITALE TOMOGRAPHIQUE EN TRANSMISSION. - Ce type de procédé connaît un très grand succès dans le domaine des rayons $X$. Le premier dispositif commercial a été mis au point par la société E.M.I. et depuis de nombreuses firmes proposent des modèles sous le sigle anglo-saxon de Scanners; on parle aussi de C.T. (computorized tomography). L'intérêt de ces techniques est de donner une information quantitative sur un paramètre. Dans le domaine des rayons $\mathrm{X}$, on dresse la carte de l'atténuation. Dans le domaine des ultrasons, on peut s'intéresser soit à l'atténuation, soit à la célérité ultrasonore.

L'hypothèse fondamentale sur laquelle repose cette technique est que la propagation d'un faisceau d'exploration collimaté se fait en ligne droite. C'est une hypothèse parfaitement justifiée pour les rayons $X$. Par contre, dans le domaine ultrasonore, c'est certainement une mauvaise approximation. Les derniers
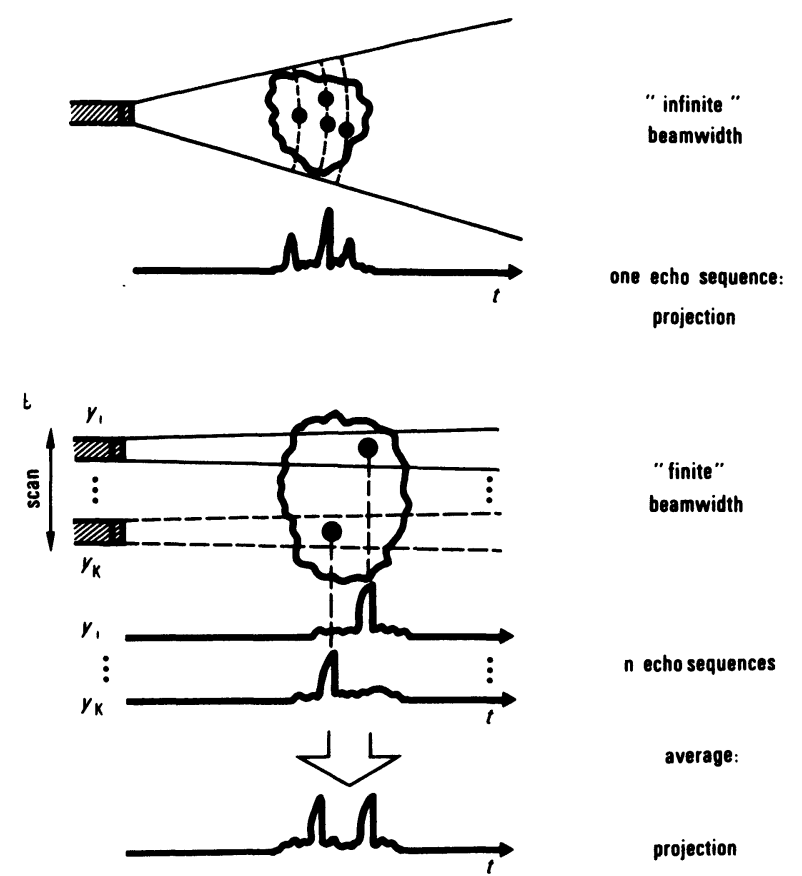

a)

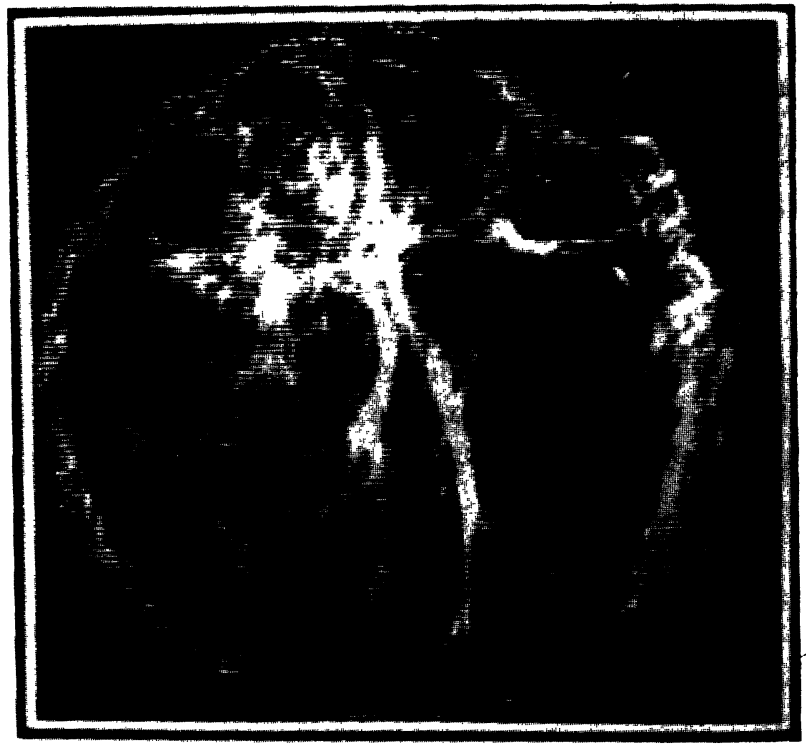

b)

Fig. 29. - a) Modes de balayage en échotomographie. En haut : utilisation d'une onde sphérique à l'émission. On obtient à chaque tir ultrasonore une projection circulaire. En bas : le transducteur est collimaté. La largeur de son faisceau est beaucoup plus petite que l'objet à examiner. Un balayage mécanique et une opération de moyenne des $n$ séquences échographiques donnent une projection linéaire. Ces projections sont mesurées pour différents angles d'incidence $\theta$ autour de l'objet. b) Reconstruction de la coupe d'un sein (carcinome).

[a) Scanning modes of echo tomography. - Spherical transmit wave. One echo sequence forms a projection (above). - Collimated transducer, with a beam-width smaller than the object. Lateral scanning and averaging of $n$ echos sequences form the projection. These projections are measured for each rotation angle $\theta$ around the object. b) Image reconstruction of breast (carcinomia).] 
raffinements de ces techniques consistent à corriger ces effets.

Pour réaliser de telles coupes tomographiques, il faut disposer d'une source d'ultrasons délivrant un faisceau collimaté et d'un détecteur d'ultrasons situé en regard de cette source à distance fixe. L'objet d'étude est immergé dans de l'eau, dans laquelle le couple sourcedétecteur peut se déplacer.

Le plan de l'objet dont on veut dresser la carte d'atténuation $\alpha(x, y)$ est divisé mathématiquement en cellules élémentaires repérées par les indices $i$ et $j$. La carte d'atténuation à déterminer se présentera sous la forme d'un tableau bidimensionnel $\alpha_{i j}$ [46].

Un faisceau d'ultrasons collimaté subira du fait de l'atténuation une décroissance exponentielle

$$
I=I_{0} \exp \left(-\int \alpha(x, y) \mathrm{d} l\right)
$$

où $I_{0}$ est l'intensité incidente, $I$ est l'intensité mesurée par le détecteur et $\alpha(x, y)$ est l'atténuation des tissus le long du trajet du faisceau.

A chaque tir ultrasonore, on obtiendra une valeur pour $I$ qui dépend bien entendu de l'origine et de la direction du faisceau dans le plan de l'objet. Deux paramètres $\theta$ et $k$ sont nécessaires pour préciser cette direction et cette origine. $k$ est un indice lié à la position de la source et $\theta$ est un indice qui dépend de l'angle du faisceau avec une direction de référence. En modifiant $N$ fois chacun des paramètres $k$ et $\theta$, les faisceaux balayent l'objet à étudier sous tous les points de vue. On obtient alors un tableau de $N^{2}$ valeurs pour $I(\theta, k)$. Il existe une relation simple entre cette matrice $I(\theta, k)$ et la matrice des $\alpha_{i j}$. En négligeant l'atténuation ultrasonore de l'eau, on obtient

$$
I(\theta, k)=I_{0} \exp \left(-\sum_{i, j \in(\theta, k)}^{N} \alpha_{i j} L_{i j}\right)
$$

$L_{i j}$ est la longueur du trajet parcouru par le faisceau repéré par $(\theta, k)$ le long de la cellule $(i j)$. Il existe de nombreux algorithmes capables d'inverser cette équation matricielle, dont le plus célèbre est celui de Radon.

Une méthode identique permet d'obtenir la carte des célérités ultrasonores $V_{i j}$ en mesurant, non pas l'intensité acoustique à la sortie du détecteur, mais en mesurant le temps de vol nécessaire pour parcourir le trajet de longueur $D$. On obtient alors, en tenant compte de la vitesse de propagation dans l'eau $(c)$, et des vitesses $V_{i j}$ une relation qui donne le temps de vol en fonction de $k$ et de $\theta$ (Fig. 30)

$$
T(\theta, k)=\sum_{i, j \in(\theta, k)}^{n} \frac{1}{V_{i j}} L_{i j}-\frac{1}{c}\left(D-\sum_{i, j \in(k, \theta)}^{n} L_{i j}^{\prime}\right) .
$$

Cette relation est, elle aussi, inversible, et différents types d'algorithmes ont été développés dans ce but [47]. On peut remarquer ici que, pour ces deux techniques, les valeurs de $I(\theta, k)$ et de $T(\theta, k)$ pour un angle d'inci-

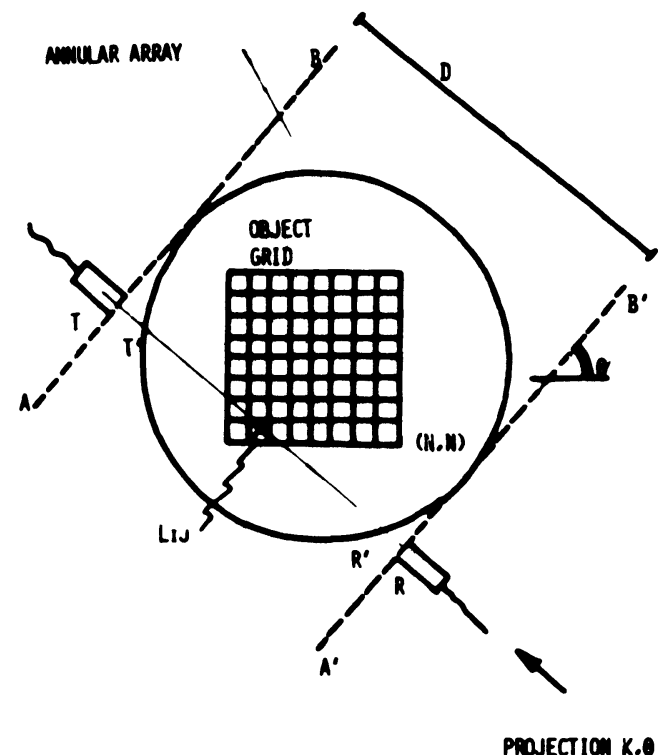

Fig. 30. - Géométrie d'un dispositif de tomographie en transmission.

[Geometry of tomographic record.]

dence $\theta$ fixé représentent deux fonctions du paramètre $k$, qui ne sont rien d'autre que les projections de la carte d'atténuation d'une part et de la célérité ultrasonore d'autre part. Parmi les algorithmes de reconstruction, les algorithmes de projection inverse, que nous avons évoqués au chapitre 5, sont particulièrement efficaces et peu bruiteux. Ils peuvent être mis en œuvre en utilisant soit des techniques de convolution, soit en réalisant des transformations de Fourier bidimensionnelles [48, 49].

Il faut reconnaître que, jusqu'à présent, les techniques de mesure d'atténuation ont donné des résultats décevants. Par contre, la cartographie des célérités ultrasonores semble plus intéressante. De nombreux travaux ont été effectués sur le sein qui est un organe pour lequel les techniques d'imagerie en transmission s'appliquent bien (Fig. 31). Des images prometteuses ont été obtenues par Greenleaf et son équipe [50].

La recherche dans ce domaine s'oriente actuellement vers la réalisation de dispositifs multitransducteurs, permettant une acquisition rapide des données expérimentales. Clément [51] a mis au point, par exemple, une barrette annulaire formée de 450 transducteurs, couvrant un champ de $360^{\circ}$ et fonctionnant à $3,5 \mathrm{MHz}$. Une commutation électronique doit permettre l'enregistrement de 10000 données en moins de 2 secondes.

Parallèlement à cette recherche technologique, l'amélioration des algorithmes de reconstruction est à l'ordre du jour. Le problème fondamental étant bien entendu lié à la validité des deux formules précédemment décrites, qui tiennent compte d'une propagation ultrasonore en ligne droite. En appliquant une telle hypothèse, on obtient alors une cartographie des vitesses qui, si elles présentent des fluctuations importantes, est certainement fausse. Plusieurs auteurs 


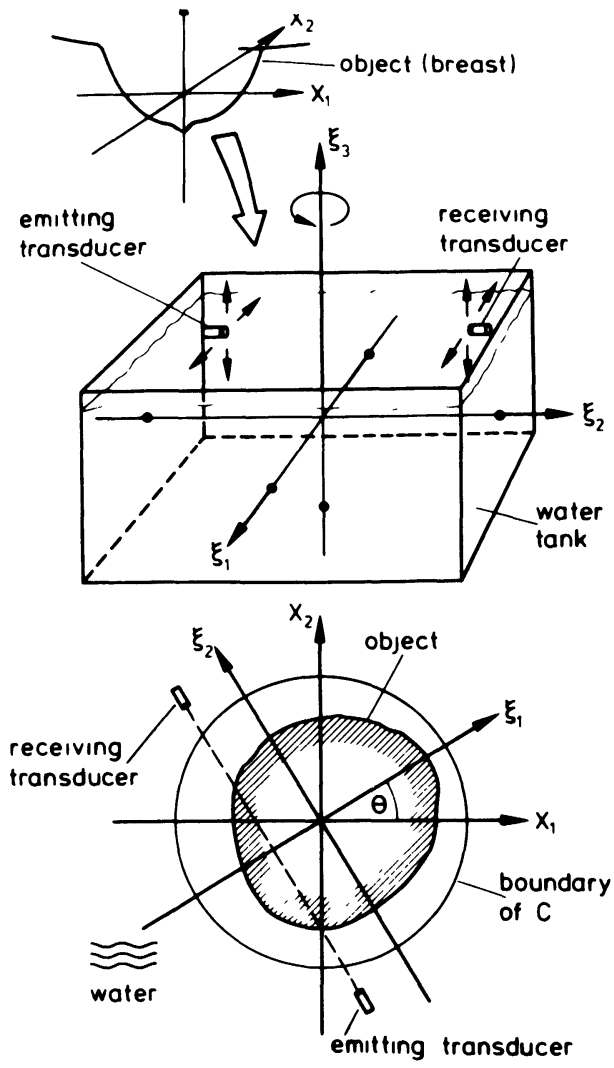

Fig. 31. - Schéma d'un dispositif de tomographie utilisé pour l'exploration du sein.

[Tomographic breast imaging system.]

ont proposé des procédés d'itération, afin d'améliorer ces images [52]. On néglige dans un premier temps les phénomènes de réfraction; on en déduit une cartographie approchée des vitesses. On trace alors les chemins parcourus par les ultrasons en tenant compte de la réfraction, ces chemins sont alors utilisés pour effectuer une deuxième opération de reconstruction tomographique etc...

Une autre méthode a été mise au point par Schomberg [53], qui fait appel à un traitement non linéaire de reconstruction.

En contrôle non destructif, les techniques de mesure de célérité ultrasonores semblent intéressantes, dans la mesure où elles doivent permettre de remonter aussi à une cartographie des contraintes résiduelles existant dans certains matériaux. Ces contraintes modifient en effet localement la vitesse du son (élasticité non linéaire) et une telle cartographie serait très utile [54].

\section{Annexe 1.}

Les principes mathématiques de l'imagerie. - Dans le cas de l'approximation de Born, le champ acoustique diffracté $p_{1}(\mathbf{r}, t)$ par l'objet, dont on cherche à dresser l'image, est relié au champ incident $p_{0}(\mathbf{r}, t)$ à travers une équation linéaire :

$$
p_{1}(\mathbf{r}, t)=S(\mathbf{r}) p_{0}(\mathbf{r}, t) .
$$

Les propriétés d'invariance de cette équation permettent de trouver une solution sous la forme d'une équation de convolution portant sur les variables d'espace et de temps.

$$
\begin{aligned}
p_{1}(\mathbf{r}, t) & =S(\mathbf{r}) p_{0}(\mathbf{r}, t) \underset{\mathbf{r}, t}{\otimes} g(\mathbf{r}, t) \\
& =\int_{\mathbf{r}_{0}} \int_{t_{0}} S\left(\mathbf{r}_{0}\right) p_{0}\left(\mathbf{r}_{0}, t_{0}\right) g\left(\mathbf{r}-\mathbf{r}_{0}, t-t_{0}\right) \mathrm{d} \mathbf{r}_{0} \mathrm{~d} t_{0} .
\end{aligned}
$$

L'effet de chaque source ponctuelle peut être mis en évidence à travers le terme $g\left(\mathbf{r}-\mathbf{r}_{0}, t-t_{0}\right)$, fonction de Green de l'équation qui n'est rien d'autre que l'onde de pression $p_{1}$ engendrée par une source ponctuelle située en $r_{0}$ et excitée par le champ incident au temps $t_{0}$

$$
\square g\left(\mathbf{r}-\mathbf{r}_{0}, t-t_{0}\right)=\delta\left(\mathbf{r}-\mathbf{r}_{0}\right) \delta\left(t-t_{0}\right) .
$$

La théorie de la diffraction [1] nous apprend que dans un espace libre cette fonction peut se mettre sous la forme

ou

$$
\begin{gathered}
g\left(\mathbf{r}-\mathbf{r}_{0}, t-t_{0}\right)=\frac{1}{4 \pi R} \delta\left(t-t_{0}-\frac{R}{c}\right) \\
R=\left|\mathbf{r}-\mathbf{r}_{0}\right| .
\end{gathered}
$$

C'est le choix classique de la solution en potentiel retardé de l'équation d'onde qui exprime le fait que chaque point source crée une perturbation qui se propage à la vitesse $c$ sous la forme d'un front d'onde sphérique divergent. La recherche de l'opération de convolution inverse qui permettra de retrouver l'ensemble des termes sources $S(\mathbf{r})$ peut être simplifiée par les remarques suivantes :

a) D'une part, il est inutile de connaître $p_{1}(\mathbf{r}, t)$ dans tout l'espace. Le principe d'Huygens et ses diverses formulations nous apprennent en effet que la valeur du champ $p_{1}(\mathbf{r}, t)$ sur un plan $\Pi$ quelconque situé en dehors de la zone source suffit à déterminer $p_{1}$ en tous points du demi-espace $z>z_{\pi}$. Une telle constatation permet alors de se ramener à une investigation spatiale bidimensionnelle, limitée à l'exploration d'un unique plan $\Pi$ (de coordonnées $x, y, z=0$ )

$$
S(\mathbf{r}) p_{0}(\mathbf{r}, t)=\left.p_{1}(\mathbf{r}, t)\right|_{z=0} \underset{x, y, t}{\otimes} g^{-1}(x, y, t)
$$

b) D'autre part, l'opération qui consiste à remonter du champ $p_{1}(x, y, t)$ aux différents termes sources peut se concevoir comme un véritable retournement de la marche du temps, qui ramènerait chacun des fronts d'ondes divergents à sa source initiale.

Cette opération de déconvolution doit associer, à la " signature " du front d'onde divergent $g\left(\mathbf{r}-\mathbf{r}_{\mathbf{0}}\right.$, $\left.t-t_{0}\right)$ sur le plan $\Pi$, la distribution $\delta\left(\mathbf{r}-\mathbf{r}_{0}\right) \delta\left(t-t_{0}\right)$.

Cette transformation peut se concevoir comme analogue à l'opération qu'on affecte en balayant le plan $\Pi$ par un transducteur focalisant, taillé en forme de coupelle sphérique de dimension infinie et de rayon de courbure égale à $z_{0}$ (la coordonnée de profondeur de $\mathbf{r}_{\mathbf{0}}$ ). Un tel transducteur, par la correction sphérique 
de retard et par l'intégration spatiale du champ acoustique sur son ouverture, délivre en effet un signal maximum chaque fois que sur son axe se présente une source à la distance $z_{0}$. Mathématiquement, ces deux opérations peuvent s'écrire sous la forme d'une convolution portant sur les trois variables $x, y, t$ par la distribution.

$$
g^{-1}(x, y, t)=I_{x} \cdot I_{y} \cdot \delta\left(t+\frac{\sqrt{x^{2}+y^{2}+z_{0}^{2}}}{c}\right) .
$$

L'image acoustique de l'objet étudié est alors reliée à $p_{1}(x, y, t)$ par la formule

$$
\begin{aligned}
S\left(\mathbf{r}^{\prime}\right) p_{0}\left(\mathbf{r}^{\prime}, t^{\prime}\right) & =\int_{x} \int_{y} p_{1}\left(x, y, t^{\prime}+\right. \\
& \left.+\frac{\sqrt{\left(x-x^{\prime}\right)^{2}+\left(y-y^{\prime}\right)^{2}+z^{\prime 2}}}{c}\right) \mathrm{d} x \mathrm{~d} y .
\end{aligned}
$$

Le choix du signal d'illumination $p_{0}\left(\mathbf{r}^{\prime}, t^{\prime}\right)$ influe sur la qualité de l'image. En mode échographique, la meilleure résolution axiale sera obtenue pour des signaux brefs. Quant au choix de la dépendance spatiale de $p_{0}\left(\mathbf{r}^{\prime}, t^{\prime}\right)$, on peut utiliser des ondes planes, des ondes sphériques ou des ondes cylindriques. En tenant compte de la dépendance spatiale de $p_{0}\left(\mathbf{r}^{\prime}, t^{\prime}\right)$ on pourra alors évaluer $S\left(x^{\prime}, y^{\prime}, z^{\prime}\right)$. Dans le cas particulier d'une illumination idéale par un front d'onde plan infiniment bref $\delta\left(t^{\prime}-z^{\prime} / c\right)$, on peut alors remplacer $t^{\prime}$ par $z^{\prime} / c$ dans les deux membres de l'équation précédente et on obtient alors une reconstitution parfaite de $S\left(x^{\prime}, y^{\prime}, z^{\prime}\right)$ selon :

$$
\begin{aligned}
S\left(x^{\prime}, y^{\prime}, z^{\prime}\right)= & \int_{x} \int_{y} p_{1}\left(x, y, \frac{z^{\prime}}{c}+\right. \\
& \left.+\frac{\sqrt{\left(x-x^{\prime}\right)^{2}+\left(y-y^{\prime}\right)^{2}+z^{\prime 2}}}{c}\right) \mathrm{d} x \mathrm{~d} y
\end{aligned}
$$

Dans les cas réels, la durée du signal d'illumination et l'ouverture finie du plan d'observation limitent la résolution spatiale.

\section{Annexe 2.}

L'holographie acoustique. - En holographie acoustique on choisit d'illuminer l'ensemble de l'objet par un signal monochromatique de pulsation $\omega$ fixée.

L'intérêt apparent d'un tel signal est lié avant tout à ses propriétés d'invariance qui entraînent qu'en tous les points d'observation on retrouve toujours la même structure monochromatique, dont la connaissance complète ne nécessite que deux paramètres : l'amplitude $A(x, y)$ et la phase $\phi(x, y)$, qui peuvent être obtenues en acoustique par un décodage temporel relativement simple (par exemple : 2 opérations de détection synchrone en quadrature fournissant respectivement $A \cos \phi$ et $A \sin \phi$ ). L'holographie acoustique repose sur l'utilisation d'une telle illumination monochromatique.

On sait que dans ce cas la perturbation créée par une source ponctuelle située en $\mathbf{r}_{0}$ peut s'écrire sous la forme

$$
p_{1}(\mathbf{r}, t)=\operatorname{Re}\left(P_{1}(\mathbf{r}) \exp (-j \omega t)\right)
$$

où $P_{1}$ vaut

$$
P_{1}(\mathbf{r})=\frac{\exp (j k R)}{4 \pi R}
$$

$\operatorname{avec} R=\left|\mathbf{r}-\mathbf{r}_{0}\right|$ et $k=\omega / c$.

Un objet quelconque soumis à une irradiation monochromatique engendrera alors un champ acoustique :

$$
p_{1}(\mathbf{r})=S(\mathbf{r}) \underset{\mathbf{r}}{\otimes} \frac{\exp (j k r)}{4 \pi r}
$$

Il faut remarquer qu'en éclairage monochromatique l'information temporelle est alors remplacée par une information de phase. La forme spatio-temporelle :

$$
\frac{1}{4 \pi R} \delta\left(t-t_{0}-R / c\right)
$$

qui était associée à chacune des sources en éclairage impulsionnel est ici réduite à la forme spatiale :

$$
\frac{\exp (j k R)}{4 \pi R}
$$

La reconnaissance de chacun des points sources se fait alors à travers la signature en phase de la perturbation $\left(5^{\prime \prime}\right)$ qu'il réémet.

On sait qu'une telle loi de phase se simplifie dans le cas de l'approximation de Fresnel où l'on peut remplacer $R$ par $z_{0}+\left(\left(x-x_{0}\right)^{2}+\left(y-y_{0}\right)^{2}\right) / 2 z_{0}$. La phase $\phi=k R$ s'écrit alors à une constante près selon la forme quadratique

$$
\phi \propto \alpha\left(\left(x-x_{0}\right)^{2}+\left(y-y_{0}\right)^{2}\right) \quad \text { où } \quad \alpha=\frac{k}{2 z_{0}} .
$$

Restituer l'ensemble des termes sources revient alors à effectuer point par point une correction quadratique de phase en $\exp \left(-j \alpha\left(x^{2}+y^{2}\right)\right)$, ce qui n'est rien d'autre qu'une transformation de Fresnel.

$$
S(\mathbf{r})=P_{1}(x, y) \underset{x, y}{\otimes} \exp \left(-j \alpha\left(x^{2}+y^{2}\right)\right) .
$$

Cette opération peut être effectuée de façon digitale en utilisant des algorithmes de transformée de Fourier rapide à deux dimensions.

\section{Annexe 3.}

Technologie des barrettes de transducteurs. - Les réseaux de transducteurs utilisés en imagerie sont des réseaux de petites sources acoustiques. Chacune de ces 
sources (éléments transducteurs) est généralement modélisée comme un petit élément vibrant en mode piston (la surface du transducteur en contact avec l'extérieur se soulève en bloc). Dès que la densité des éléments transducteurs devient importante, la réalité devient beaucoup plus complexe. Il apparaît alors, d'une part un couplage important entre éléments et, d'autre part la vibration de chaque transducteur se fait alors suivant plusieurs modes. Ces phénomènes peuvent modifier de façon très sensible la réponse impulsionnelle du transducteur qui peut durer beaucoup plus longtemps. Ils modifient aussi très fortement le diagramme de directivité d'un élément, qui au lieu d'être pratiquement omnidirectionnel, devient relativement étroit avec en outre l'apparition de lobes secondaires extrêmement intenses. Ces modifications de la réponse impulsionnelle temporelle et du diagramme de directivité spatiale du transducteur élémentaire sont très préjudiciables à la qualité des images. La résolution axiale des images échographiques qui dépend de la durée des échos peut être fortement dégradée, et la résolution latérale qui dépend des procédés de focalisation ou de reconstruction est modifiée par l'allure du diagramme de directivité élémentaire. En outre, dans les techniques de balayage sectoriel électronique (phased array) et dans celles de synthèse de faisceaux obliques (beam forming), il est nécessaire, pour obtenir des ouvertures angulaires importantes, d'avoir des transducteurs élémentaires pratiquement omnidirectionnels.

A. Le premier problème est celui de la réponse impulsionnelle temporelle de l'élément transducteur. Elle doit être la plus brève possible. On doit éviter la génération de tous les modes de vibration possédant un facteur de qualité $Q$ élevé. La philosophie générale est de travailler si possible en mode piston. L'amortissement de ce mode peut être réalisé en couplant efficacement l'énergie de ce mode avec le milieu de propagation à étudier. On peut utiliser pour cela une ou plusieurs fenêtres d'adaptation acoustique positionnées sur la face avant du transducteur. Des configurations comportant 2 lames d'épaisseurs $\lambda / 4$, d'impédance correctement choisie [55-57] permettent d'obtenir des réponses impulsionnelles très brèves à 6 , 20 et $40 \mathrm{db}$.

- On peut aussi réaliser un amortissement des transducteurs par la face arrière, en utilisant des milieux arrière d'impédance acoustique proche de celle $\mathrm{du}$ transducteur piézoélectrique. On obtient ainsi d'excellents résultats mais avec une perte de sensibilité de l'ordre de $20 \mathrm{db}$ comparée à la technique précédente.

- A côté de ce mode piston (mode en épaisseur), il existe pour des transducteurs de petite dimension d'autres modes parasites qu'on cherche à éliminer :

- Le mode de dilatation est un mode transverse de facteur $Q$ élevé où l'énergie acoustique effectue des aller-retour entre les parois transverses du transducteur. On peut augmenter la fréquence de ce mode pour le filtrer électriquement, en diminuant la dimension transverse du transducteur élémentaire.

- Il existe un autre mode important qui est celui de l'oscillateur harmonique formé d'une part de la masse du transducteur et d'autre part du ressort équivalent à la face arrière élastique sur laquelle repose le transducteur [58]. La fréquence de ce mode peut être très proche de celle du mode piston, et son facteur de qualité est très élevé, ce qui peut entraîner dans certains cas des traînées de la réponse impulsionnelle à $50 \mathrm{db}$ de l'ordre de $50 \mu \mathrm{s}$. Les premiers centimètres des images échographiques sont alors fortement dégradés. Des solutions ont été proposées pour éliminer ce mode, en utilisant des configurations symétriques en face avant et arrière [58].

B. Les modifications du diagramme de directivité du transducteur élémentaire ont deux origines bien distinctes : le couplage électrique et le couplage acoustique inter-éléments.

- Le couplage d'origine électrique est associé aux capacités parasites inter-éléments qui sont souvent du même ordre de grandeur que la capacité d'un élément. Les lignes de champ électrique sont déformées et une des solutions à ce problème est de séparer les transducteurs élémentaires par un fort rainurage du milieu piézoélectrique [59].

- Le couplage d'origine acoustique est dû à un certain nombre d'ondes de surface qui peuvent prendre naissance aux différentes interfaces $[60,61]$.

A l'interface entre le milieu piézoélectrique et la face arrière des ondes de Rayleigh peuvent se propager. Ce sont des ondes dont l'amplitude décroît exponentiellement avec la profondeur dans la face arrière. Une solution pour les éliminer est de rainurer cette face

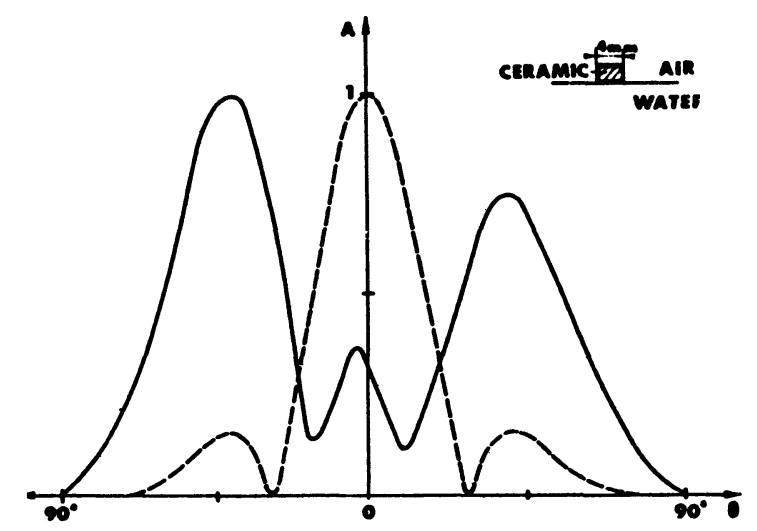

Fig. 32. - Effet des modes de Lamb sur le diagramme de directivité d'un transducteur élémentaire. La largeur de l'élément est de $4 \mathrm{~mm}$, la fréquence de $800 \mathrm{kHz}$. - Sur la figure en ligne pointillée : diagramme théorique du transducteur vibrant en mode piston. - Sur la figure pleine : diagramme expérimental présentant deux lobes intenses.

[Resonance effects induced by lamb waves. Characteristic radiation pattern. - Dotted line : calculated directivity pattern of a piston mode. - Full line : measured directivity pattern.] 
arrière sur une profondeur suffisante, et avec un pas de rainurage inférieur à la demi-longueur d'onde de ces ondes [62].

- On peut aussi observer dans le matériau piézoélectrique (céramique) et dans les lames de la face avant des ondes de Lamb. Ce sont des modes guidés qui apparaissent dans des plaques de faible épaisseur. Ces ondes de Lamb qui se déplacent relativement vite comparées aux ondes longitudinales circulant dans l'eau, peuvent générer dans l'eau des ondes parasites dont la direction avec la normale à la barrette fait un angle $\theta$ égal à $\pm \arcsin \left(c / c_{\mathrm{L}}\right), c_{\mathrm{L}}$ étant la célérité de l'onde de Lamb considérée [63]. Le diagramme de direc- tivité du transducteur élémentaire est alors complètement modifié et il apparaît généralement deux lobes secondaires symétriques très intenses, qui dégradent fortement la qualité de la focalisation (Fig. 32).

On peut éliminer efficacement ce type d'onde en découpant la face avant sur une profondeur suffisante avec un pas inférieur à la demi-longueur d'onde des modes de Lamb qui peuvent apparaître aux fréquences considérées [64].

En tenant compte de tous ces paramètres, on peut obtenir des barrettes de bonne qualité. Les réalisations japonaises et celles de la firme Hewlett Packard en sont de bons exemples.

\section{Bibliographie}

[1] Morse, P. M., Ingard, K. J., Theoretical Acoustics (New York, Mac Graw Hill) 1968, p. 319.

[2] Jones, H. W. et Williams, C. J., Lenses and ultrasonic imaging, Acoustical Holography (Plenum) 7 (1978) 133-153.

[3] Clayden, R. T., Brown, P. H., A simple, high definition imaging system for the location and inspection of submerged objects in turbid conditions. Proceedings of the Oceanology International-78, Brighton Conference, Mars 1978.

[4] Green, P. S., Schaeffer, L. F., Jones, P. D., SuArez, J. R., A new high performance ultrasonic camera. Acoustical Holography (Plenum) 5 (1974) 493-503.

[5] Sutron, J. L., A tutorial on underwater acoustic imaging. Acoustical Imaging (Plenum) 9 (1980) 599-630.

[6] Maguer, P., Gelly, J. F. et Grall, G., An underwater focused acoustic imaging system. Acoustical Imaging (Plenum) 10 (1982) 607-618.

[7] LEITH, E. N., UPATNIEKs, J., Reconstructed wavefronts and communication theory. J. Opt. Soc. Am. 52 (1962) 1123-1130.

[8] Clark, A. V., On obtaining maximum performance from liquid surface levitation holography. Acoustical Imaging (Plenum) 8 (1979) 417-453.

[9] Alais, P., Real time acoustical imaging with a $256 \times 256$ matrix of electrostatic transducers. Acoustical Holography (Plenum) 5 (1974) 671-684.

[10] Swartz, R. G. et Plummer, Monolithic silicon PVF2 piezoelectric arrays for ultrasonic imaging. Acoustical Imaging (Plenum) 8 (1979) 69, 95.

[11] Collins, H. D., Gribble, R. P., Hall, T. E., Lechelt, J., Acoustical holography matrix array imaging system for the underwater inspection of offshore oil platform weldments. Acoustical Imaging (Plenum) 8 (1979) 97-117.

[12] Das, P., White, G. A., Sinma, B. K., Lanzl, C., Tiersten, M. F. et MCDonald, J. L., Ultrasonic imaging using monolithic mosaic transducer utilizing trapped energy modes. Acoustical Imaging (Plenum) 8 (1979) 119-135.

[13] Nitadori, K., Mano, K. et Kamata, H., An experimental underwater acoustic imaging system using multibeam scanning. Acoustical Imaging (Plenum) 8 (1979) 249-266.

[14] Koppelmann, R. F., Keating, P. N., Three dimensional acoustic imaging. Acoustical Imaging (Ple. num) 8 (1979) $26 \overline{6}-280$.
[15] Sutton, J. L., Thorn, J. V., Booth, N. O. et Saltzer, B. A., Description of navy holographic underwater acoustic imaging system. Acoustical Imaging (Plenum) 8 (1979) 219-234.

[16] Hildebrand, B. P. et Doctor, S. R., Alternative scanning geometries for high speed imaging with linear ultrasonic arrays. Acoustical Imaging (Plenum) 9 (1980) 1-22.

[17] Collins, H., Hall, T. C. et Wilson, R. L., Focused line source linear array holography using a three dimensional computer reconstruction system. Acoustical Imaging (Plenum) 9 (1980) 39-56.

[18] Torguet, R., Bruneel, C., Bridoux, E., Rouvaen, J. M. et Nongaillard, B., Ultrafast echotomographic system using optical processing of ultrasonic signals. Acoustical Holography (Plenum) 7 (1977) 79-85.

[19] Bruneel, C., Nongaillard, C., Torguet, R., BriDOUX, E., RouvaEN, J. M., Reconstruction of an acoustical image using an acousto-electronic lens device. Ultrasonic 15 № 6 (1977) 263.

[20] Havlice, J. F., Kino, G. S., Kofol, J. S. et QUATE, C. F., Electronically focused acoustic imaging device. Acoustical Holography (Plenum), vol. 5, 1974, p. 317-334.

[21] Young, J. W., Electronically scanned and focused receiving array. Acoustical Holography (Plenum), vol. 7, 1977, p. 387-403.

[22] Burckhardt, C. B., HoffmanN, H. and GrandСНАмР, P. A., The ultrasound axicon : a device for focusing over a large depth. J. Acoust. Soc. Amer. 54 (1973) 1628-1630.

[23] Foster, F. S., Patterson, M. S., Arditi, M. et HUNT, J. W., The conical scanner : a two transducer ultrasound scatter imaging technique. Ultrasonic Imaging 3 (1981) 62-82.

[24] Auphan, M., Les transducteurs à réseau annulaire focalisant en poursuite d'échos. Acta Electronica 22 (1979) 119-127.

[25] Marich, K. W., Ramsey, S. D., Wilson, D. A., Holzemer, J. F., Burch, D. J., Taenzer, J. C. et GREEN, P. S., An improved medical ultrasonic imaging system for scanning peripheral anatomy. Ultrasonic Imaging 3 (1981) 309-322.

[26] Arditi, M., Taylor, W. B., Foster, F. S. et HUNT, J. W., An annular array system for high resolution breast echography. Ultrasonic Imaging 4 (1982) 1-31. 
[27] Kubota, J., IschiI, J. et SASAKI, S., High resolution ultrasonic testing system using dynamic focusing and signal correlation. A paraître, Acoustical Imaging 11.

[28] HASSLER, D., HöNIG, D. et SchwaRz, R., Ultrasound B scanner with multiline array. Ultrasonic Imaging 4 (1982) 32-43.

[29] ANDERson, W. A., A new real time phased array sector scanner for imaging the entire adult human heart. Ultrasound in Medicine 3B (1977) 1547-1558.

[30] SOMER, J. C., Electronic sector scanning for ultrasound diagnos. Ultrasonics 6 (1968) 153-159.

[31] Thurstone, F. L. et von Ramm, O. T., Electronic beam steering for ultrasonic imaging 2 nd world congress on ultrasonics in medicine, RotterdamNetherlands, Excerpta Medica, Amsterdam (1974) p. 43-48.

[32] Karrer, H. E., Dias, J. F., Larson, J. D. et Pering, R., A phased array acoustic imaging system for medical use. Acoustical Imaging (Plenum) 10 (1982) 47-64.

[33] Manes, G. F., Susini, C., Portoli, P. et Atzeni, C., New processing technique in ultrasound imaging system. Acoustical Imaging (Plenum) 10 (1982) 177-194.

[34] Gehlbach, S. et Alvarez, R. E., Digital ultrasound imaging techniques using vector sampling and raster line reconstruction. Ultrasonic Imaging 3 (1981) 83-107.

[35] Perroneau, P. A., Bournat, J. P., Bufnon, A., BARBET, A. et XHAARD, M., Theoretical and practical aspect of pulsed Doppler flowmeter, cardiovascular applications of ultrasound, R. S. (Reneman, North Holland, American Elsevier) 1974.

[36] Gerzberg, L. et Meindl, J., Power spectrum centroid detection for Doppler systems applications, Ultrasonic Imaging 2 (1981) 232-261.

[37] FInK, M. et HotTIER, F., Short time Fourier analysis and diffraction effect in biological tissue characterization, Acoustical Imaging (Plenum) 12 (1982) 493-503.

[38] Corl, P., Kino, G. S., Desilets, C. S. et Grant, P. M., A digital synthetic focus acoustic imaging system. Acoustical Imaging (Plenum) 8 (1979) 39-54.

[39] Bennett, S., Peterson, D. K., Corl, D. et Kino, G. S., A real time synthetic aperture digital acoustic imaging system. Acoustical Imaging (Plenum) 10 (1982) 669-692.

[40] NorTon, S. J. et Linzer, M., Ultrasonic reflectivity tomography : Reconstruction with circular transducer arrays. Ultrasonic Imaging 1 (1979) 154-184.

[41] Liang, K., KhuRi-Yakub, T., Chou, C. M. et KINo, G. S., A three-dimensional synthetic focus system. Acoustical Imaging (Plenum) 10 (1982) 643-668.

[42] Rose, J. H. et Krumhansl, A., Technique for determining flow characteristics from ultrasonic scattering amplitudes. Proc. ARPA/AFML Rev. of Progr. in Quant. NDE, juillet 1979, La Jolla, Californie, Air Force Report.

[43] Bleistein, N. et Cohen, J. K., Application of inverse method to non destructive evaluation of flaws. Semi-Annal Report (Rockwell International Science Center, Thousand Oaks, Californie) p. 112.
[44] Miller, D. et ERMERT, H., Tomographic reconstruction of B. Scan Images. Acoustical Imaging (Plenum) 10 (1982) 347-364.

[45] Maderlechner, G., Hundt, E., Kronmüller, E. et Trautenberg, E., Experimental results of computerized ultrasound echotomography. Acoustical Imaging (Plenum) 10 (1982) 415-426.

[46] Greenleaf, J. F., Johnson, S. A., Lee, S. L., HerMAN, G. T. and Wood, E. H., Algebraic reconstruction of spatial distributions of acoustic absorption within tissue from their 2-D acoustic projections. Acoustical Holography (Plenum) 5 (1974) 591-603.

[47] Greenleaf, J. F., Johnson, S. A., Samoyoa, W. F. and Duck, F. A., Algebraic reconstruction of spatial distributions of acoustic velocities in tissue from their time of flight profiles. Acoustical Holography (Plenum) 6 (1975) 71, 90.

[48] MerCEREAU, R. M., Recovering multidimensional signals from their projection. Computers Graphics and Image Processing 1 (1973) 179-195.

[49] Ramachandran, G. N. et LaKShminarayanan, A. V., $3 D$ reconstruction from radiographs and electron micrographs; II : applications of convolution instead of Fourier transforms. Proc. Nat. Acad. Sci. 68, $\mathrm{n}^{\circ} 9$ (1971) 2236-40.

[50] Greenleaf, J. F., Kenue, S. K., Rajagopalan, B., BAHN, R. C. et Johnson, S. A., Breast imaging by ultrasonic computer assisted tomography. Acoustical Imaging (Plenum) 8 (1980) 599-614, A. Metherell.

[51] Clement, M., Alais, P., Roucayrol, J. C., Perrin, J., Computerized ultrasonic tomography by electronic scanning and steering of a ring array. Acoustical Imaging (Plenum) 10 (1982) 397-413, P. Alais.

[52] Johnson, S. A., Greenleaf, W. F., Samayoa, W. F., DucK, F. A. et SJostrand, J. D., Reconstruction of $3 D$ velocity fields and other parameters by acoustic Ray tracing. 1975 Ultrasonic Symposium Proceeding I.E.E.E., Cat. No 75, p. 46-51, CHP 994 ISU, 1975.

[53] SCHOMBERG, H., Non linear image reconstruction from ultrasonic time-of-flight projections. Acoustical Imaging (Plenum) 10 (1982) 381-396.

[54] Hildebrand, B. P. et Hufferd, D. E., Computorized reconstruction of ultrasonic velocity fields for mapping of residual stress. Acoustical Holography (Plenum) 7 (1977) 245-262.

[55] Lewis, G. K., A matrix technique for analysing the performance of multilayered front matched and bached piezoelectric ceramic transducers. Acoustical Imaging (Plenum) 8 (1979) 395-416.

[56] Desilets, C. S., Fraser, J. D. et Kino, G. S., The Design of Efficient Broad-Band Piezoelectric Transducers, Trans. Son. Ultrason. SU 25, № 3 (1978).

[57] Souquet, J., Defranould, P. et Desbois, J., Design of Low-Loss Wide-Band Ultrasonic Transducers for Noninvasive Medical Application, Trans. Son. Ultrason. SU 26 № 2 (1979).

[58] LARSON, J. D., A new vibrational mode in tall narrow piezoelectric elements. 1979 Ultrasonics Symposium Proceedings, I.E.E.E. $79 \mathrm{CH}$ 148-95 U, p. 108-113.

[59] Bruneel, C., Delannoy, B., Torguet, R., BriDOUX, E., Lasota, H., Electrical coupling effects in an ultrasonic transducer array. Ultrasonic 17 No 6 (1979) 255-260. 
[60] Pappalardo, M., Hybrid linear and matrix acoustic arrays, Ultrason. (1981) 81.

[61] Gelly, J. F. et Maerfeld, C., Properties for a $2 D$ Multiplexed Array for Acoustic Imaging, Proceedings of IEEE Ultrasonics Symposium, 1981.

[62] Hanafy, A., Dead zone elimination in acoustic arrays. Ultrasonic Imaging 2 (1980) 302-312.
[63] Delannoy, B. et Bruneel, C., Origin of anomalous behavior in transducers used in acoustical imaging. Acoustical Imaging (Plenum) 10 (1982) 65-81.

[64] LARSON, J. D., Non ideal radiators in phased array transducers. 1981 Ultrasonics Symposium Proceedings I.E.E.E. p. 673, 684. 